\title{
Non-Gaussian Analysis of Turbulent Boundary Layer Fluctuating Pressure on Aircraft Skin Panels
}

\author{
Alexander Steinwolf* \\ University of Auckland, Auckland 1001, New Zealand \\ Stephen A. Rizzi ${ }^{\dagger}$ \\ NASA Langley Research Center, Hampton, Virginia 23681-2199, USA
}

The purpose of the study is to investigate the probability density function (PDF) of turbulent boundary layer fluctuating pressures measured on the outer sidewall of a supersonic transport aircraft and to approximate these PDFs by analytical models. Experimental flight results show that the fluctuating pressure PDFs differ from the Gaussian distribution even for standard smooth surface conditions. The PDF tails are wider and longer than those of the Gaussian model. For pressure fluctuations in front of forwardfacing step discontinuities, deviations from the Gaussian model are more significant and the PDFs become asymmetrical. There is a certain spatial pattern of the skewness and kurtosis behavior depending on the distance upstream from the step. All characteristics related to non-Gaussian behavior are highly dependent upon the distance from the step and the step height, less dependent on aircraft speed, and not dependent on the fuselage location. A Hermite polynomial transform model and a piecewise-Gaussian model fit the flight data well both for the smooth and stepped conditions. The piecewise-Gaussian approximation can be additionally regarded for convenience in usage after the model is constructed.

\section{Nomenclature}

a $=$ coefficient in the Pearson Type IV, Johnson $S_{u}$, and Hermite polynomial PDFs

$B \quad=$ parameter of the symmetric piecewise-Gaussian PDF

$b=$ coefficient in the Pearson Type IV, Johnson $S_{u}$, and Hermite polynomial PDFs

C $=$ scale coefficient of the Pearson Type IV, Hermite polynomial, and piecewise-Gaussian PDFs

$C \quad=$ crest factor of experimental time history record

$f(v)=$ Hermite polynomial transform function

$H=$ vertical shift of the central section of the symmetric piecewise-Gaussian PDF

$\mathrm{He}_{j}(v)=$ Hermite polynomials

$h_{j} \quad=$ Hermite polynomial coefficients

$k=$ order of truncation of the Hermite polynomial transform

$M_{n} \quad=$ probability distribution central moment of $n$-th order

$m \quad=$ mean value of random process or probability distribution

$N \quad=$ number of points in experimental time history records

$P(u) \quad=$ probability density function (PDF)

$P_{A}(u) \quad=$ asymmetric piecewise-Gaussian PDF

\footnotetext{
* Senior Lecturer, Department of Mechanical Engineering, University of Auckland, Private Bag 92019, Auckland, New Zealand, a.steinwolf@auckland.ac.nz (corresponding author).

Aerospace Engineer, Structural Acoustics Branch, Mail Stop 463, stephen.a.rizzi@nasa.gov, AIAA Associate Fellow.
} 


\begin{tabular}{|c|c|}
\hline$P_{G}(u)$ & $=$ Gaussian PDF \\
\hline$P_{G C}(u)$ & $=$ Gram-Charlier PDF \\
\hline$P_{H}(u)$ & $=$ Hermite polynomial PDF \\
\hline$P_{S}(u)$ & $=$ symmetric piecewise-Gaussian PDF \\
\hline$P_{S 2}(u, \sigma, \beta)$ & $=$ symmetric Type 2 piecewise-Gaussian PDF \\
\hline$P_{S u}(u)$ & $=$ Johnson $S_{u} \mathrm{PDF}$ \\
\hline$P_{I V}(u)$ & $=$ Pearson Type IV PDF \\
\hline$Q$ & $=$ scale coefficient of the symmetric piecewise-Gaussian PDF \\
\hline$Q_{A}$ & $=$ scale coefficient of the asymmetric piecewise-Gaussian PDF \\
\hline$q$ & $=$ coefficient in the Hermite polynomial PDF \\
\hline$\Delta t$ & $=$ time increment \\
\hline$u$ & $=$ variable of the PDF function \\
\hline$\tilde{u}$ & $=$ standardized non-dimensional variable \\
\hline$X_{1,2}(u)$ & $=$ components of the symmetric piecewise-Gaussian PDF \\
\hline$x(t)$ & $=$ time history of the boundary layer fluctuating pressure \\
\hline Y & $=$ parameter of the symmetric piecewise-Gaussian PDF \\
\hline$\alpha$ & $=$ non-Gaussian parameter of the asymmetric piecewise-Gaussian PDF \\
\hline$\beta$ & $=$ non-Gaussian parameter of the piecewise-Gaussian PDF \\
\hline$\gamma$ & $=$ kurtosis of probability distribution \\
\hline$\gamma^{*}$ & $=$ experimental kurtosis value \\
\hline$\varepsilon$ & $=$ coefficient in the Johnson $S_{u} \mathrm{PDF}$ \\
\hline$\zeta$ & $=$ coefficient in the Pearson Type IV PDF \\
\hline$\eta$ & $=$ coefficient in the Johnson $S_{u}$ PDF \\
\hline$\lambda$ & $=$ skewness of probability distribution \\
\hline$\lambda *$ & $=$ experimental skewness value \\
\hline$\mu$ & $=$ argument of the vertex point of asymmetric piecewise-Gaussian PDF \\
\hline $\pm v$ & $=$ argument values where two sections of the symmetric piecewise-Gaussian PDF meet \\
\hline $\pm v_{L, R}$ & $=$ argument values where sections of the asymmetric piecewise-Gaussian PDF meet \\
\hline$\sigma$ & $=$ standard deviation of random process or probability distribution \\
\hline$\sigma_{1,2}$ & $=$ parameters of the symmetric piecewise-Gaussian PDF \\
\hline$\sigma_{1 L, 2 L, 1 R, 2 R}$ & $=$ parameters of the asymmetric piecewise-Gaussian PDF \\
\hline
\end{tabular}

\section{Introduction}

Vibration of aircraft skin panels is a matter of concern for structural failures and is a cause of passenger cabin noise in commercial transports. The fluctuating pressures associated with the turbulent boundary layer is one of several sources which excite fuselage sidewall and wing panels. This loading mechanism exists in both subsonic and supersonic flight conditions. The panel response is accentuated in the supersonic case because of coupling between the fluctuating pressure and flexible panel deflections [1]. As fully coupled computations for prediction of boundary layer induced vibration are at present not feasible for practical applications, the emphasis has been on development of rigid wall pressure models.

Turbulent boundary layer pressure fluctuations during cruise at a constant speed and altitude are commonly regarded as random stationary process. The power spectral density (PSD) typically characterizes this random excitation in the absence of fluctuating pressure time histories. Several theoretical and experimentally based power spectrum models have been developed for smooth wall conditions $[2,3,4]$ and then extended to the case of the stepped wall conditions based on wind tunnel measurements [5] and flight test data [6]. However, the PSD approach describes the time history fully only if the random process under consideration is Gaussian. If the process is 
non-Gaussian, some time history features are not reflected in PSD and, thus, other measures such as the probability density function (PDF) are additionally required along with the power spectrum. The PDF characteristics of turbulent boundary layer pressure fluctuations on an aircraft exterior have not been extensively investigated.

If an excitation, particularly the fluctuating pressure, appears to be different from the Gaussian random model, then the dynamic system response, particularly of the skin panel, will be non-Gaussian as well. This can have significant implications with regard to two issues:

1) the acoustic radiation from vibrating fuselage panels into the aircraft interior becomes a non-Gaussian noise which may be different in its influence on passengers;

2) random cyclic stress/strains induced in the skin panel itself will contribute to material fatigue differently than would be the case if they were Gaussian random processes.

The second problem refers to sonic fatigue of high-speed aircraft structures that usually results from elevated acoustic loads near the engine effluxes, where overall sound pressure levels often exceed $160 \mathrm{~dB}$. High-cycle fatigue induced by turbulent boundary layer pressure fluctuations is normally not considered a problem because of reduced overall levels (typically in the 130-135 dB range) and shorter correlation lengths relative to an acoustic excitation from engines. However, this changes dramatically if there are discontinuities on the fuselage sidewall.

It has been recently discovered with data from wind tunnel [5] and flight [6] experiments that, upstream of small forward-facing step discontinuities, the turbulent boundary layer loading can be amplified in excess of $30 \mathrm{~dB}$ compared to the smooth sidewall, and in excess of $20 \mathrm{~dB}$ downstream of aft-facing steps. Thus, near such discontinuities on exterior surfaces, e.g. skin panel joints and door edges, the turbulent boundary layer pressure excitation is such that high-cycle fatigue may become a concern.

When addressing this within the limits of the PSD approach, the dynamic response predicted may be accurate in terms of its root-mean-square level and PSD, but lacking correct information about the response probability distribution. If the fluctuating pressure excitation and, as a result, the skin panel response are erroneously assumed to have a Gaussian PDF, then the well-known spectral-based cycle counting techniques $[7,8,9]$ may lead to an inaccurate fatigue life prediction. The relation between the probability distribution of instantaneous values of the fluctuating pressure considered in this study and the PDF of stress/strain cyclic ranges governing accumulation of fatigue damage in a structural component is as follows.

If the fluctuating pressure excitation is non-Gaussian, the analysis of a skin panel response should consist of two stages. Initially, the stress response will be characterized by a non-Gaussian PDF of instantaneous values. This first stage is necessary because the general statistical dynamics theory (as spectral analysis in the Gaussian case) operates with the instantaneous value characteristics of the input and output, not with the cyclic ranges. It will be the next stage of the analysis to determine how the PDF of cyclic ranges changes when the PDF of instantaneous stress values is non-Gaussian. For example, it is well known that for narrowband random processes, the Gaussian distribution of instantaneous values generates a Rayleigh distribution of peaks [7]. By analogy, it has been shown that for certain non-Gaussian instantaneous stress PDF expressed in a piecewise form, the corresponding PDF of stress peaks may be presented in a piecewise-Rayleigh form [10].

Currently, the second stage of random loading fatigue analysis is beyond the scope of this work which concentrates on the fluctuating pressure PDF of instantaneous values considered at the first stage of finding the structural response. However, the correlation between effects of non-Gaussian behavior on both kinds of probability distributions is obvious. What is particularly addressed in this paper is that since the essential part of the fatigue damage results from the high stress cyclic ranges, any non-Gaussian difference in the instantaneous value distribution tails is especially important.

The characterization of PDFs of the turbulent boundary layer pressure fluctuations is required so that accurate probability distributions can be used in subsequent structural fatigue and noise radiation calculations. The main purpose of the present study is the PDF analysis of experimental fluctuating pressure signals over a broad range of flight and fuselage surface conditions. Another objective is to explore and further develop analytical expressions capable of modeling the PDFs of fluctuating pressure signals if they appear to be non-Gaussian. When comparing various probability distribution models, the ease with which they may be used in the subsequent analysis of dynamics and fatigue is considered along with the accuracy of modeling.

\section{Flight Experiment}

Experimental data acquired from flight tests of the Tupolev Tu-144LL Supersonic Flying Laboratory [11, 12] were used in this study. For subsonic and supersonic flight conditions, long contiguous records of pressure fluctuation time history were acquired by transducers distributed over the length of the fuselage on both sides of the aircraft. 


\section{A. Smooth Sidewall Measurements.}

Aluminum window blanks (WB) were installed in place of the windows, as described in Ref [11], to serve as rigid mounting points for pressure transducers. The window blanks had a smooth external surface and were slightly curved to conform with the fuselage outer diameter. They were set strictly flush with the exterior aircraft surface. Gaps between the window blanks and the fuselage skin were filled with a gasket sealant to minimize the effects of these gaps on the turbulent boundary layer.

Kulite pressure transducers (model XCS-190-15D) for measuring pressure fluctuations were mounted through the window blanks. Details of the transducer installation can be found in Ref [11]. As the transducers should be electrically isolated from the structure in order to reduce noise in the signal, each of them was threaded into a plastic boss (threaded plug) separating the transducer from the window blank material. The sensitive element of the transducer is protected on the external side by a circular plate with small holes through which the pressure is sensed.

A flush transducer installation with the outer surface was considered critical. The thread with the finest available pitch was used on the transducer tube in order to make the adjustment of the transducer face's flushness with the outer surface precisely controllable. The transducer outer faces were also carefully sanded to be as flat as possible without compromising the strength of the connection between the protecting plate and the transducer body. Flushness measurements made over the transducer circumference indicated that at most measurement points the variation was within twenty thousandths of a millimeter.

On the starboard side of the aircraft, seven window blanks were equipped with the fluctuating pressure transducers as shown in Fig. 1. All of these transducers measured pressure fluctuations on the smooth exterior surface. For the purposes of this paper, measurements at three locations (close to the aircraft nose, in the middle cabin, and in the aft cabin) were considered. The approximate distances from the aircraft nose (including nose boom) are provided in Table 1 for the three selected transducers (N1.1, N4.1 and N7.2) which were located at the window blanks WB1, WB4 and WB7.

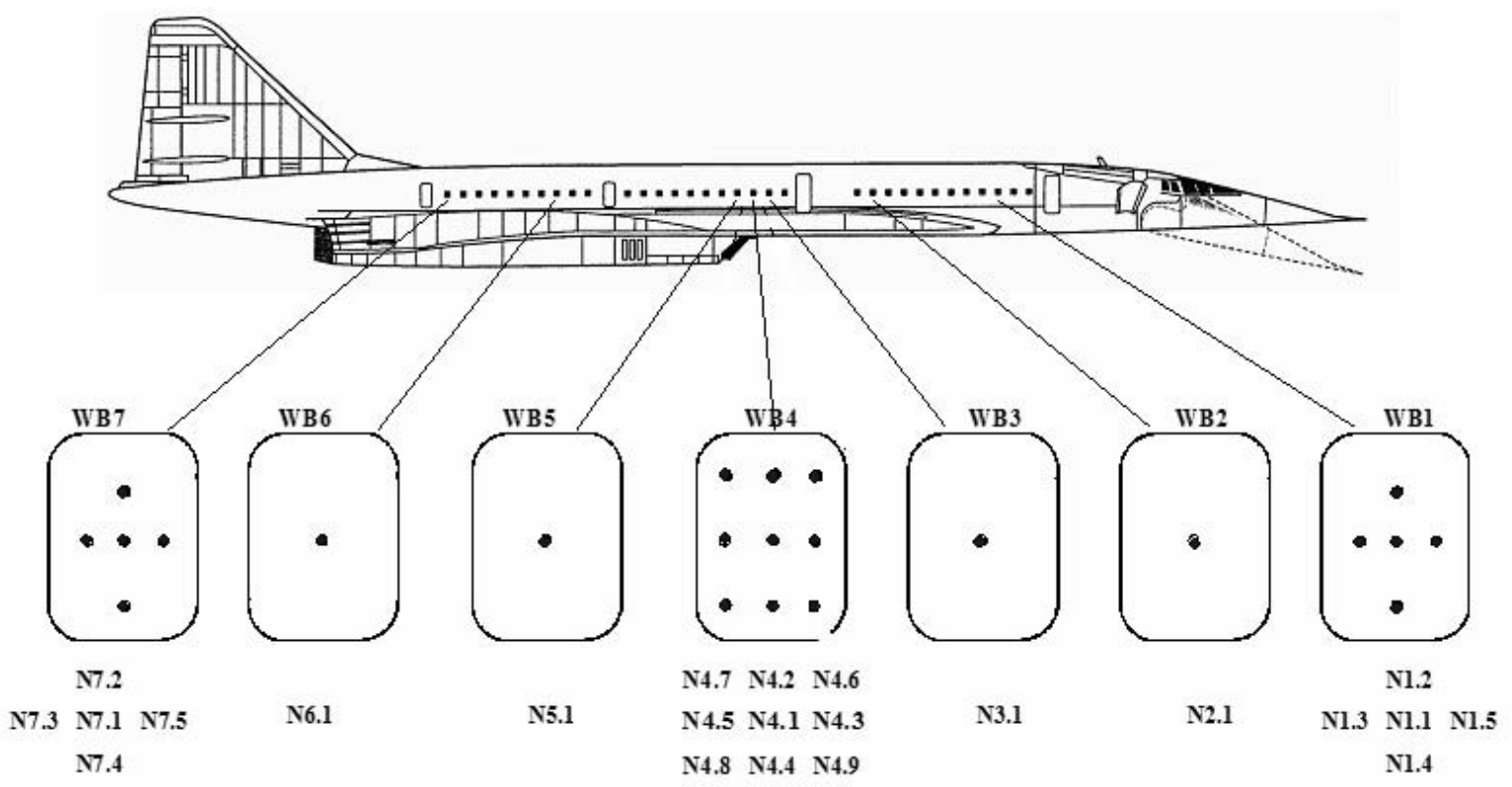

Fig 1. Location and identification of window blanks instrumented with Kulite pressure transducers on the starboard side of the Tu-144LL. 
Table 1. Distances of window blanks with transducers from aircraft nose (incl. nose boom)

\begin{tabular}{|c|c|c|c|c|c|c|}
\hline & $\begin{array}{c}\text { Surface } \\
\text { conditions }\end{array}$ & \multicolumn{2}{|c|}{ Smooth sidewall } & $\begin{array}{l}\text { Smooth } \\
\text { sidewall }\end{array}$ & \multicolumn{2}{|c|}{ Smooth sidewall } \\
\hline Starboard & $\begin{array}{c}\text { Window } \\
\text { Blank }\end{array}$ & \multicolumn{2}{|c|}{ WB 1} & WB 4 & \multicolumn{2}{|c|}{ WB 7} \\
\hline side & $\begin{array}{l}\text { Distance } \\
\text { from nose }\end{array}$ & \multicolumn{2}{|c|}{$18.9 \mathrm{~m}$} & $32.6 \mathrm{~m}$ & \multicolumn{2}{|c|}{$49.3 \mathrm{~m}$} \\
\hline \multirow{3}{*}{ Port side } & $\begin{array}{c}\text { Surface } \\
\text { conditions }\end{array}$ & $\begin{array}{c}\text { Forward } \\
\text { facing step }\end{array}$ & $\begin{array}{c}\text { Aft } \\
\text { facing step }\end{array}$ & & $\begin{array}{c}\text { Forward } \\
\text { facing step }\end{array}$ & $\begin{array}{c}\text { Aft } \\
\text { facing step }\end{array}$ \\
\hline & $\begin{array}{l}\text { Window } \\
\text { Blank }\end{array}$ & WB 8 & WB 9 & & WB 10 & WB 11 \\
\hline & $\begin{array}{l}\text { Distance } \\
\text { from nose }\end{array}$ & $21.7 \mathrm{~m}$ & $22.6 \mathrm{~m}$ & & $44.9 \mathrm{~m}$ & $45.8 \mathrm{~m}$ \\
\hline
\end{tabular}

\section{B. Sidewall Discontinuities Setup.}

To compare with the smooth sidewall results from the starboard side of the aircraft, the port side was used to investigate the effect of sidewall discontinuities [12]. For this purpose, two step-plates were installed on the fuselage exterior spanning adjacent window blanks as shown in Fig. 2. One plate provided forward-facing and aft-facing step discontinuities at the front of the aircraft and the second plate formed another identical pair of discontinuities at the rear of the aircraft.

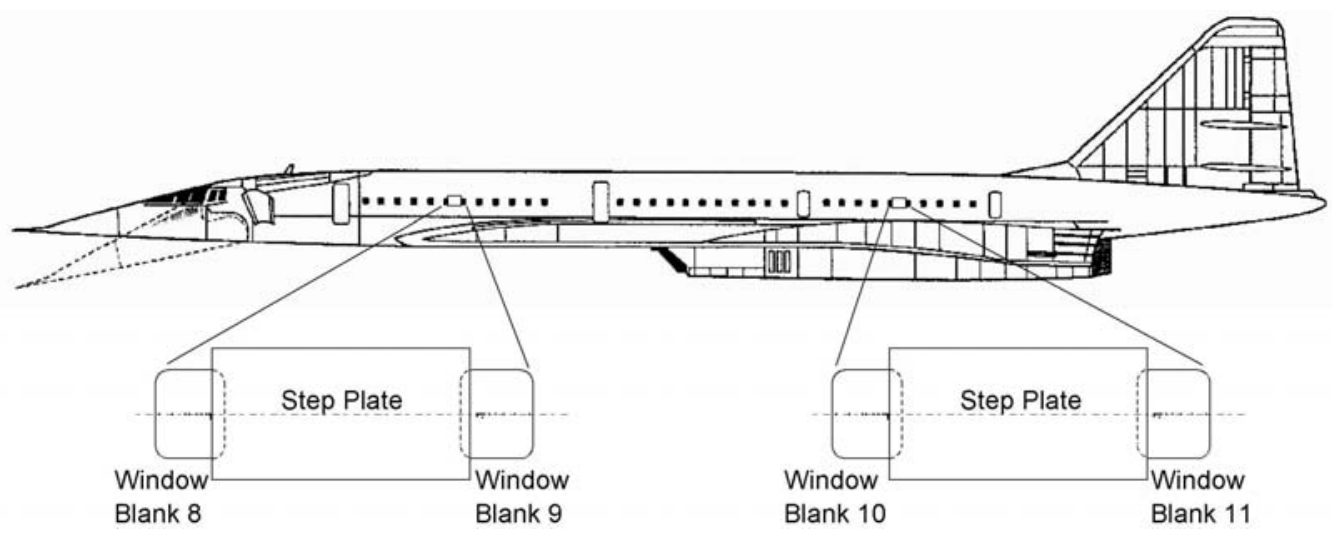

Fig 2. Location of step plates spanning adjacent window blanks on the port side of the Tu-144LL.

The locations of the plates were chosen approximately opposite to two of the smooth sidewall measurement points on the other side of the aircraft (WB1 and WB7). The forward plate between WB8 and WB9 on the port side was in a longitudinal location close to WB1 on the starboard side. Similarly, the rear plate between WB10 and WB11 was located opposite WB7. Approximate distances from the aircraft nose to all window blank centers specified on the port side (WB8-WB11) are also presented in Table 1.

The step plates consisted of two panels, 4 and $3 \mathrm{~mm}$ thick, riveted to each other. This allowed variations in the step height. With both panels in place, a total step height of $7 \mathrm{~mm}$ was achieved. When the upper $3 \mathrm{~mm}$ layer was removed, the step height was lowered to $4 \mathrm{~mm}$. The width of the lower panel was $305 \mathrm{~mm}$ and that of the upper panel was $216 \mathrm{~mm}$ [12]. Transitions from the $216 \mathrm{~mm}$ to $305 \mathrm{~mm}$ width and from the $305 \mathrm{~mm}$ width of the lower plate to the fuselage surface were made from a sculpted filler material to avoid steps in the circumferential direction. Both panels were curved to conform to the circumferential curvature at the locations of their installation.

To measure more closely spaced pressure fluctuations in vicinity of the step discontinuities, a smaller transducer was required. Miniature Kulite pressure transducers (model XCS-062-15D) with $1.63 \mathrm{~mm}$ diameter were installed [12] as shown in Fig 3. Transducers in window blanks 8 and 10 were exposed to the effects of a forward facing step, while those in window blanks 9 and 11 were exposed to the effects of an aft facing step. The XCS-062-15D transducer had nominally the same performance characteristics as the larger XCS-190-15D transducer used for smooth sidewall measurements described in previous section. 


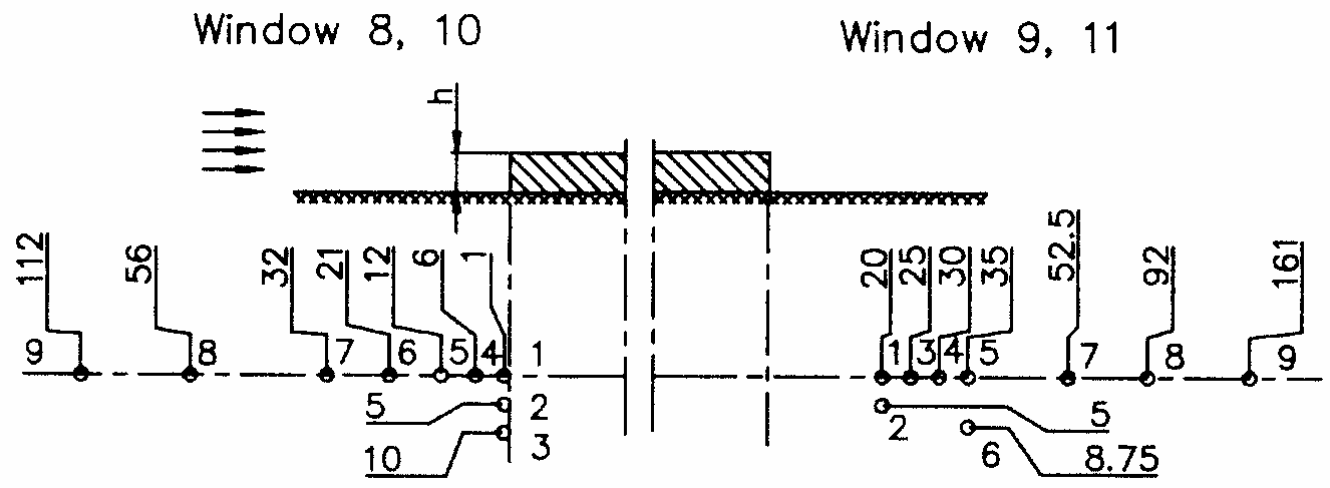

Fig. 3 Layout of transducer locations in front of forward-facing steps and behind aft-facing steps with distances in millimeters from the step edge.

As for the larger transducers, a plastic mounting boss provided electrical isolation of the smaller transducer, however these transducers were unthreaded. They were glued to the inside of the mounting boss with Hysol epoxy, flush with the boss face. A fine metric thread, M3 x 0.35, on the outside of the boss was used to set the exposed end of the boss flush with the exterior surface.

Due to the small size of the transducer, it was not possible to obtain flushness measurements over the transducer circumference as was the case for larger transducers on the starboard side of the aircraft. One measurement was taken for each of the XCS-062-15D transducers and these results are documented in Ref [12]. Generally, the same precision as for the smooth sidewall experiment (within twenty thousandths of a millimeter) was achieved for the miniature transducers in the vicinity of the step discontinuities.

\section{Data Acquisition.}

Independent signal conditioning of each pressure transducer was provided to allow for simultaneous measurements. Based upon expected levels and in order to make the most out of the dynamic range of the data recorder used, a fixed gain was programmed into each stage of the two-stage amplifier [11]. The first stage was capacitively coupled to the second stage to block the DC component of the input, which could be significant since the Kulite pressure transducers had a thin reference tube in the back, which connected a cavity behind the sensitive element with the cabin. Thus the sensitive element experienced a static pressure differential between the aircraft interior and exterior.

Only the fluctuating component of the signals was allowed to pass to the amplifier second stage and be subsequently recorded. Because the maximum output voltage from the first stage is 20 volts peak to peak, special precautions were taken not to over-range this stage with the amplified DC signal. A gain of 100 for the first amplification stage and the second stage gain of 5 were chosen to make up the total gain of 500.The second amplification stage could pass frequencies from $1 \mathrm{~Hz}$ to $50 \mathrm{kHz}$ interval.

To capture signals with the above frequencies, a Metrum RSR 512 digital tape recorder was used. Its features included 32 analog input channels, 12-bit quantization to provide $70 \mathrm{~dB}$ dynamic range, selectable input voltage ranges of $0.1 \mathrm{~V}$ to $10 \mathrm{~V}$ peak, a high speed digital output port for transferring data to a PC for post-flight data analysis, and an auto-range capability which was utilized as discussed below.

To analyze the probability density function, more attention is required to selecting the measurement range appropriately than when doing a regular frequency domain FFT processing. If a signal is recorded with carelessly low gain, there will be lack of space for the proper number of histogram intervals necessary to describe the PDF accurately. To avoid this, the dynamic range of recording was maximized through an elaborate procedure of autoranging all input signals.

First, the peak voltage on each channel is initially set to $0.1 \mathrm{~V}$ value. By starting at this most sensitive value, a low sensitivity from a previous test condition would not persist and penalize the dynamic range. Then the overranging channels had their peak voltages increased by the recorder auto-range logic until the channel no longer overranged or until the least sensitive $10 \mathrm{~V}$ value had been reached. This entire auto-range process for all channels usually took about a second. 
Because the signal time histories could have relatively rare high peaks, the second step of the auto-ranging process utilized a longer time record. In this continuous mode, each channel was set so that its peak voltage could be increased (but not decreased) if positive or negative peaks of the input signal exceeded a threshold value reached on the first step of the brief auto-ranging mode. The subsequent continuous mode could be enabled for a user selectable period, which typically was on the order of ten seconds.

Because the Metrum recorder did not have internal anti-aliasing filters, this function was performed using external filters. A Frequency Devices 5016 mainframe with plug-in filter cards (model D68L8E) had 8-pole, 6-zero elliptic low-pass filters with a $-3 \mathrm{~dB}$ fixed corner frequency of $11.2 \mathrm{kHz}$, corresponding to the upper frequency of the $10 \mathrm{kHz}$ third-octave band. The sampling rate used for all transducers was $40 \mathrm{kHz}$, setting a Nyquist frequency of $20 \mathrm{kHz}$, well above the desired cut-off frequency of $11.2 \mathrm{kHz}$.

Since the number of transducers exceeded the 32 channels available in the Metrum recorder, flight data was recorded in three banks. One bank contained pressure fluctuations on the smooth sidewall measured on the starboard side of the aircraft. Another bank contained data from window blanks 8 and 9 on the port side (the forward step plate), and a third bank contained data from window blanks 10 and 11 (the rear step plate). Following the tests, data was digitally transferred to a computer for post-processing.

Data studied in this paper were acquired from five flight conditions ( 1 subsonic and 4 supersonic), each of which with 2 step heights. Measurements at subsonic and supersonic speeds were made during horizontal flight-path segments. Calibrations of Kulite transducers were performed both before and after flights and no significant differences between them were observed.

\section{Data Analysis}

As discussed above, a common approach to turbulent boundary layer fluctuating pressure analysis is to assume that the random process under consideration is both stationary and Gaussian and can be described by its power spectral density. The first assumption that the data is stationary is justified by the fact that all flight conditions were stable during each particular recording (typically for 30-60 seconds).

The second assumption of the fluctuating pressure signal being Gaussian should be examined and, if it is not valid, the probability density function must be computed and its deviations from the Gaussian model

$$
P_{G}(u)=\frac{1}{\sigma \sqrt{2 \pi}} \exp \left[-\frac{(u-m)^{2}}{2 \sigma^{2}}\right]
$$

must be studied. The principal characters describing non-Gaussian PDF features are skewness $\lambda$ and kurtosis $\gamma$

$$
\lambda=\frac{M_{3}}{\left(M_{2}\right)^{3 / 2}}=\frac{M_{3}}{\sigma^{3}}, \quad \gamma=\frac{M_{4}}{\left(M_{2}\right)^{2}}=\frac{M_{4}}{\sigma^{4}}
$$

governed by central moments

$$
M_{n}=\int_{-\infty}^{\infty}(u-m)^{n} P(u) d u
$$

of the probability density function $P(u)$.

The Gaussian distribution (1) is specified by two parameters: mean value $m$ and variance $\sigma^{2}$, where the latter coincides with the second moment $M_{2}$. However, there is no variability in PDF moments higher than the second. The skewness and kurtosis related to the third and fourth moments are constants $(\lambda=0, \gamma=3)$ for the Gaussian PDF. Hence, any other $\lambda$ and $\gamma$ values obtained would be an indication of Gaussian model failure, i.e. a suggestion about limitations on the use of the power spectral density approach. 
If the experimental PDF is non-Gaussian, but still symmetric with respect to the mean, kurtosis is the only additional parameter required to describe deviations from the Gaussian model. The kurtosis characterizes the sharpness or flatness of the PDF peak and the wideness or narrowness of the PDF tails. A kurtosis value greater than 3 indicates a sharper peak and wider tails than in the Gaussian PDF with the same standard deviation $\sigma$. If the experimental PDF is asymmetrical with respect to the mean value $m$, then the skewness is non-zero and its sign will indicate the direction in which the PDF is skewed.

Considering the signals as stationary ergodic random processes, the mean value was calculated by time averaging

$$
m=\frac{1}{N} \sum_{i=1}^{N} x(i \Delta t)
$$

of the data records digitized with the time increment $\Delta t=0.025 \mathrm{~ms}$, corresponding to a $40 \mathrm{kHz}$ sampling rate. Then, the moment characteristics (including variance $\sigma^{2}=M_{2}$ ) and non-Gaussian moments of higher order were computed by averaging centralized time history values $[x(i \Delta t)-m]$ raised to the power equal to the moment order $n$

$$
M_{n}=\frac{1}{N} \sum_{i=1}^{N}[x(i \Delta t)-m]^{n}
$$

Finally, the central moments $M_{n}(n=2,3,4)$ obtained lead to the required skewness and kurtosis values according to Eqs (2).

For non-Gaussian data with increased kurtosis (wider PDF tails), there is another indicator of non-Gaussian behavior. This characteristic, called crest factor, is not related to the probability distribution and emerges directly from a time history. The crest factor value is a ratio between the height of the largest peak in the time history and the signal standard deviation

$$
c=\frac{x_{\max }(i \Delta t)}{\sigma}
$$

However, in contrast to the kurtosis, no strict theoretical value can be defined for the crest factor of a Gaussian process because the magnitude of the largest peak depends on the length of time history sample, even for the same random signal. Furthermore, the kurtosis summarizes the effect of all excessive peaks that make the signal non-Gaussian whereas the crest factor takes into account only one of them - the largest. Therefore, the kurtosis value is a more robust characteristic than the crest factor.

If the skewness and/or kurtosis values are indicative of non-Gaussian behavior, then more detailed analysis involving the probability density function should be carried out and the PDF obtained should be compared to the Gaussian distribution given by Eq (1). Since the step character of a histogram (a PDF estimation from data) makes comparison with the Gaussian curve difficult, the experimental PDFs are plotted below in the form of a curve connecting vertices of the histogram bins.

Whether the distribution tails are non-Gaussian cannot be visually determined on PDF graphs with regular linear scale. To analyze this effect, one needs to present the PDF on a logarithmic ordinate scale (see Figs 4,b,d,f and 5,b,d below), which magnifies differences between theoretical and experimental probabilities for high amplitude values. If the PDF is asymmetric, the left and right tails will be different.

It should be also taken into account that, the higher the time history peak, the less frequently it occurs. Hence, longer data records are necessary to have representative statistical material and to calculate the experimental PDF tails properly. In this study the number of points $N$ in a typical fluctuating pressure time history record for each of the flight conditions was 1,200,000-2,400,000 points. 


\section{Non-Gaussian Characteristics of Flight Data}

\section{A. Results for Smooth Surface with No Discontinuities.}

The data analysis techniques described in the previous section were first implemented for the fluctuating pressure records measured on the smooth fuselage sidewall. It appeared that these data for the surface with no discontinuities exhibit non-Gaussian behavior which is readily noted from the kurtosis results. For the subsonic flight at $750 \mathrm{~km} / \mathrm{h}$, the kurtosis reached a value of 4.2 compared to 3.0, that is the value inherent of Gaussian random processes.

For supersonic conditions, kurtosis decreased with increasing aircraft speed, however remained essentially different from the Gaussian value (numerical results for kurtosis are given in Section IV.D below). The variation of kurtosis along the length of the fuselage was insignificant. For each of the flight conditions, the kurtosis values reported were obtained by averaging the individual kurtosis values at three transducer locations (N1.1, N4.1, and N7.2 shown in Fig. 1).

Results of PDF computations are presented in Fig. 4. The PDF tails (see Figs. 4,b,d,f) were much wider than those of the Gaussian distribution shown by the dotted curves. In other words, the probability of a high peak occurrence in the time history of the experimental data is several times larger than that predicted by the Gaussian model. For data records of finite length, it means that the experimental record contains such high peaks that would not occur in a synthetic time history generated with the same PSD but within the limits of the Gaussian model.

The above conclusion should be taken into account for any numerical or experimental simulations of the turbulent boundary layer fluctuating pressure data, even in the case of no discontinuities on the fuselage surface. The phenomenon can be also characterized in terms of crest factor (6). It reached a value of $c=10$ for the subsonic and $C=9$ for the supersonic flight conditions. By comparison, the crest factor for records of similar length for a Gaussian process would be roughly $4.5-5.0$.

In the PDF central area, non-Gaussian behavior was mild (see Figs. 4,a,c,e). The peak of the experimental PDFs of pressure fluctuation signals is somewhat taller and sharper than that of the Gaussian distribution, presented by the dotted curve. There is no observed PDF asymmetry for the data from the smooth surface, neither at the tails nor in the central PDF section. This is confirmed by the skewness values obtained, which were negligible for all subsonic and supersonic flight conditions. However, the situation changes dramatically if the sidewall surface is not smooth.

\section{B. Probability Density Functions for the Surface with Forward-Facing Step Discontinuities.}

The qualitative difference between the fluctuating pressure data obtained from the smooth surface and the surface with discontinuities is that upstream of the forward-facing steps the PDF becomes essentially asymmetrical with respect to the mean value. It means that equal positive and negative pressure values may have different probabilities of their occurrence in a particular point of aircraft exterior. That is what is not described by the frequency spectrum approach and gives rise to the non-Gaussian skewness value.

Two typical skewed PDFs are shown in Fig. 5 where experimental data (solid curves) are presented in comparison with the Gaussian model (dotted curves). One of these PDFs is for the fluctuating pressure measured immediately in front of the forward-facing step. At this location the PDF peak is shifted from zero in the negative direction (Fig. 5,a) making the left and right slopes of the PDF curve different. This difference is aggravated at the PDF tails (Fig. 5,b) when the right tail is much longer and lays well above the Gaussian curve. The left tail of the experimental PDF is shorter and positioned fully under the Gaussian curve.

If the non-Gaussian PDF of such a shape is substituted as a function $P(u)$ into Eq (3) with $n=3$, then the higher probability at the right tail, i.e. larger $P(u)$ values for positive arguments, will lead to some positive value of the third moment $M_{3}$ and finally to a positive skewness $\lambda$ according to Eq (2). For example, the particular skewness value obtained for the $7 \mathrm{~mm}$ step at Mach 2.0 was $\lambda=1.2$. The corresponding PDF is shown in Fig. 5,a,b. The above fluctuating pressure PDF behavior was observed in all measurements by the transducer closest $(1 \mathrm{~mm})$ to the forward-facing step, for both step heights and all supersonic speeds.

However, an opposite situation with negative skewness occurred further upstream of the step. This was detected by the transducers located either at $32 \mathrm{~mm}$ or $56 \mathrm{~mm}$ before the step depending on the step height, for aircraft speeds Mach 1.6 and greater. The second PDF example shown in Figs. 5,c,d is for the $4 \mathrm{~mm}$ step and Mach 2.0 at the $32 \mathrm{~mm}$ distance, where the largest negative skewness $(\lambda=-1.3)$ was found. Contrary to the point close to the step, the PDF peak is now shifted in the positive direction (compare Fig. 5,a with Fig. 5,c) and the PDF tails swapped their positions, with the left tail becoming wider than the right (compare Fig. 5,b with Fig. 5,d). 


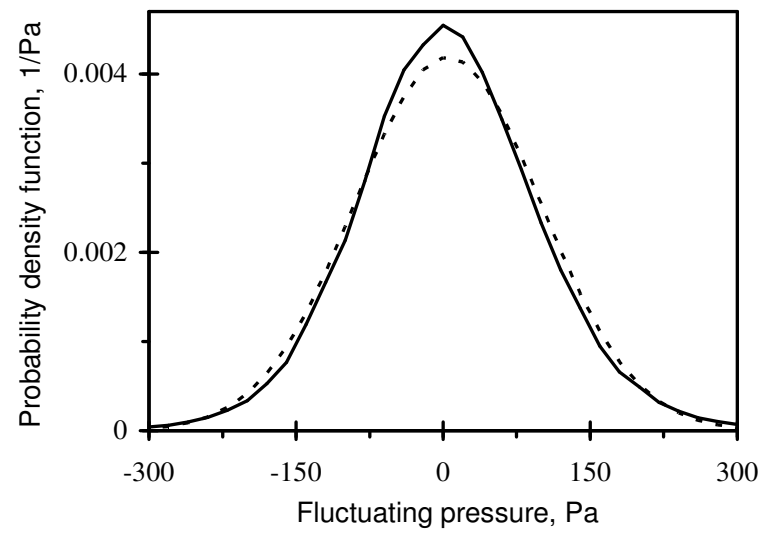

a) PDF peak for $600 \mathrm{~km} / \mathrm{h}$

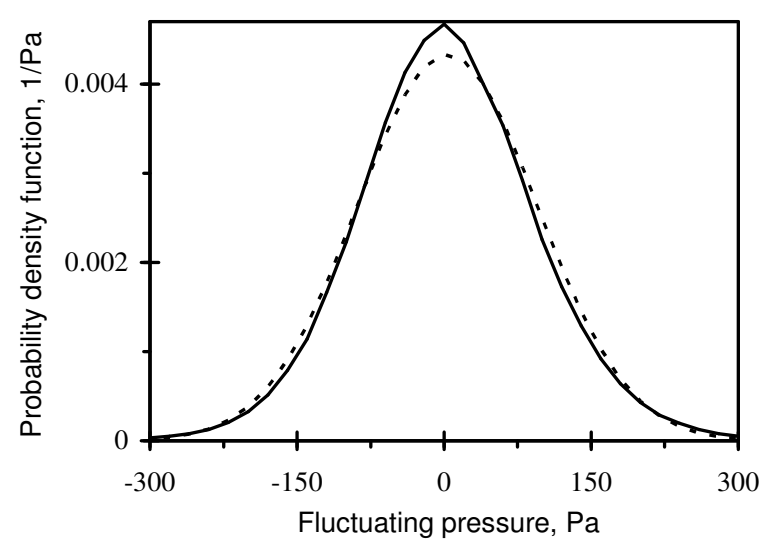

c) PDF peak for Mach 1.2

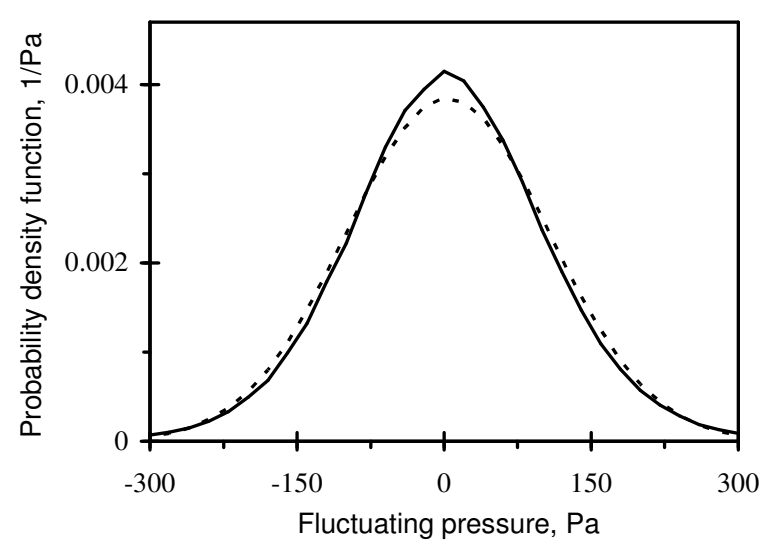

e) PDF peak for Mach 2.0

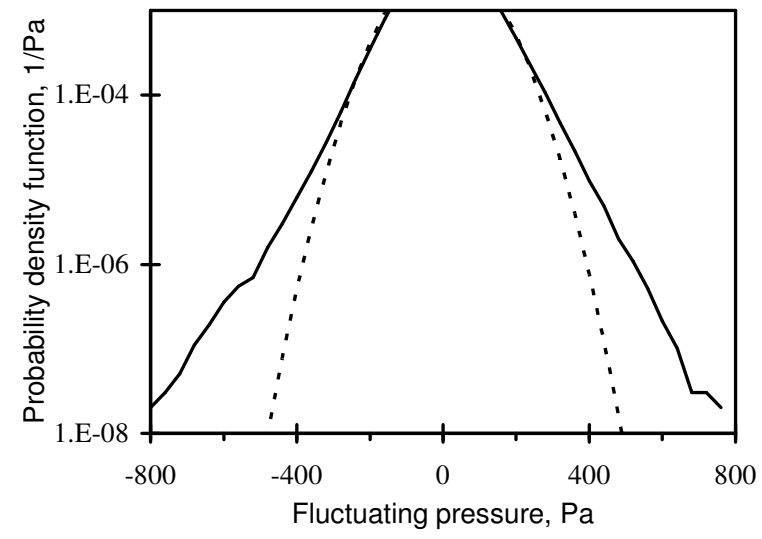

b) PDF tails for $600 \mathrm{~km} / \mathrm{h}$

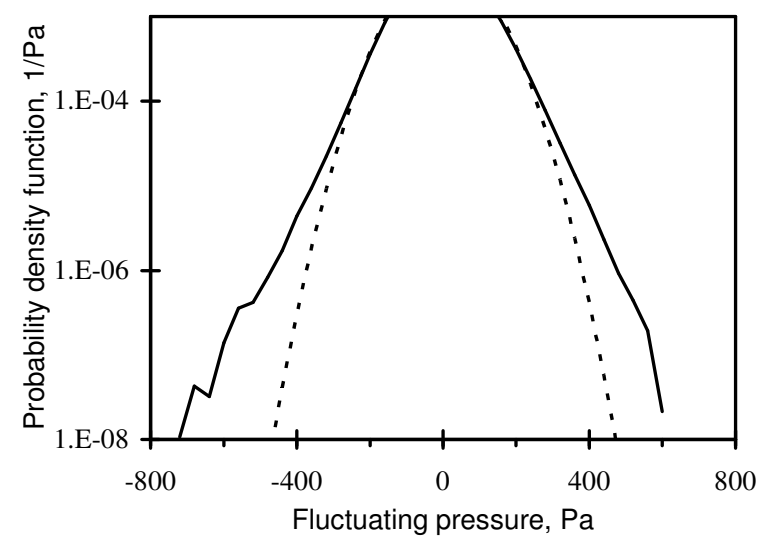

d) PDF tails for Mach 1.2

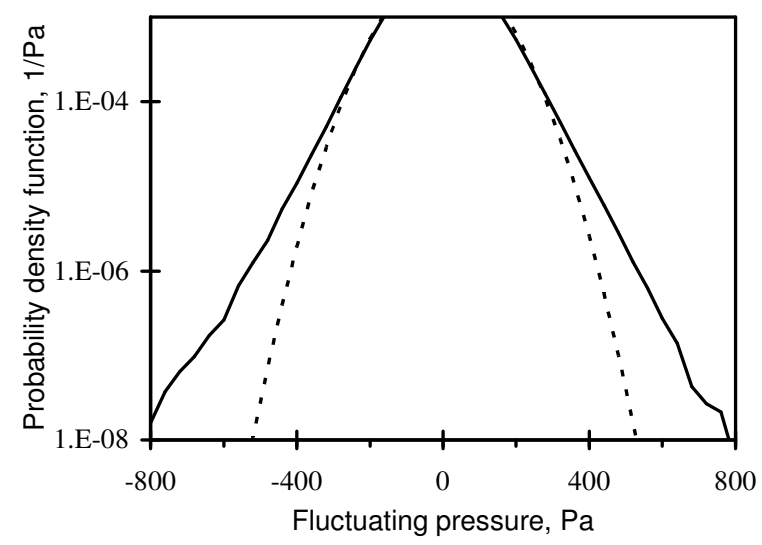

f) PDF tails for Mach 2.0

Fig. 4 Probability density functions of smooth wall fluctuating pressure (solid) and the Gaussian model (dotted) 


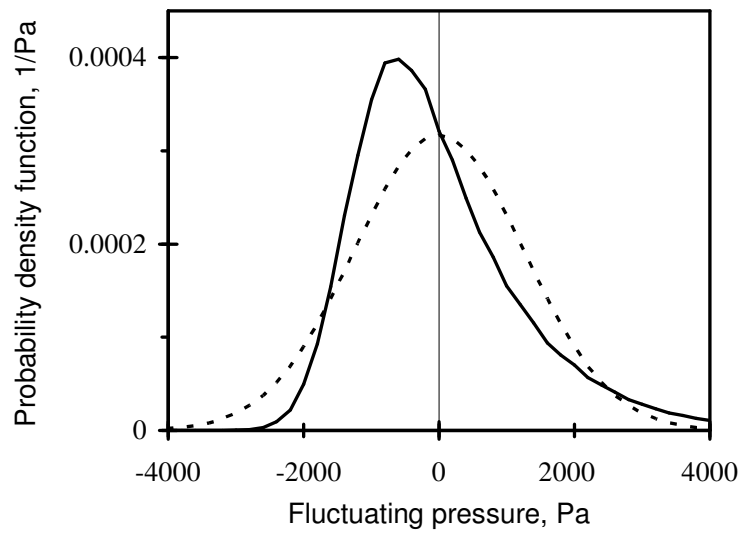

a) PDF peak at a location immediately before the step (skewness $\lambda=1.2$ )

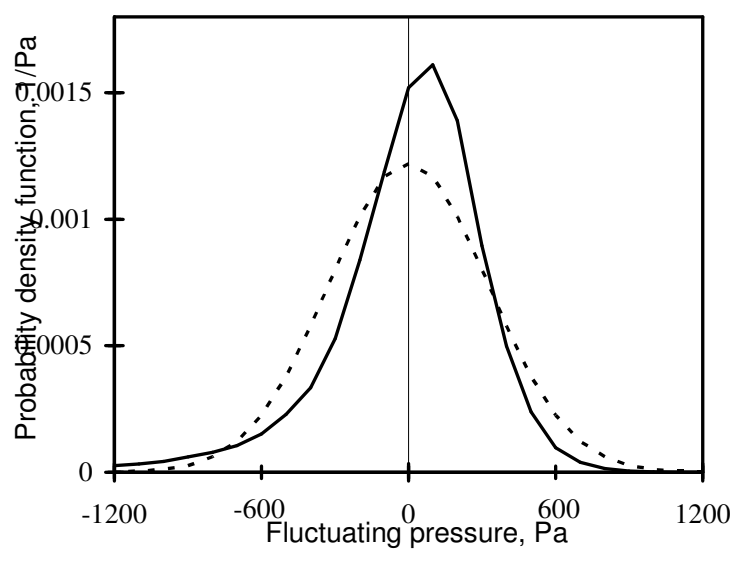

c) PDF peak at a location further from the step (skewness $\lambda=-1.3$ )

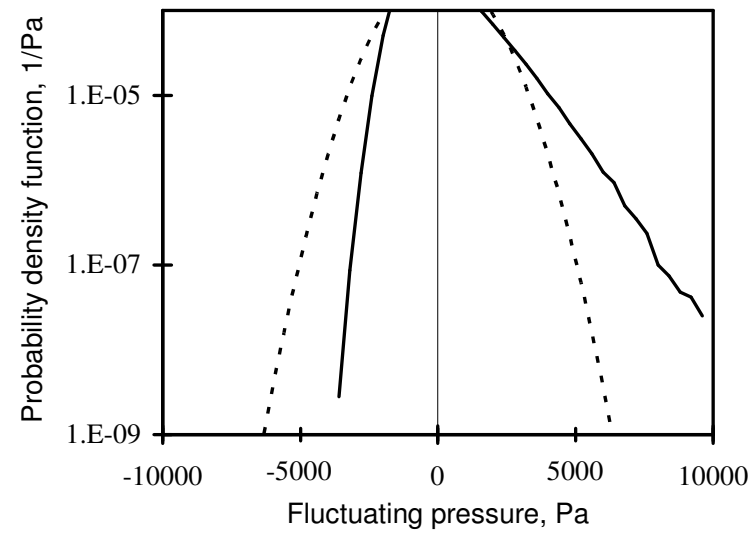

b) PDF tails at a location immediately before the step (skewness $\lambda=1.2$ )

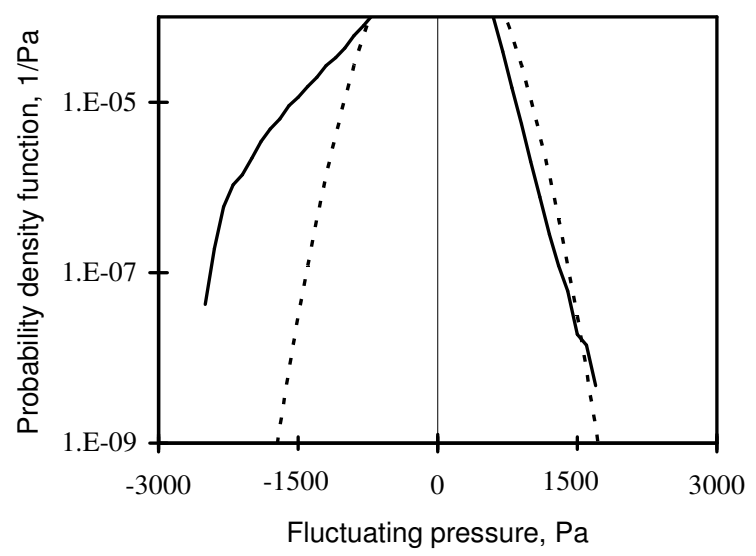

d) PDF tails at a location further from the step (skewness $\lambda=\mathbf{- 1 . 3}$ )

Fig. 5 Probability density functions of fluctuating pressure at two locations upstream of the forward-facing step (flight data at Mach 2 - solid curves, Gaussian model - dotted curves)

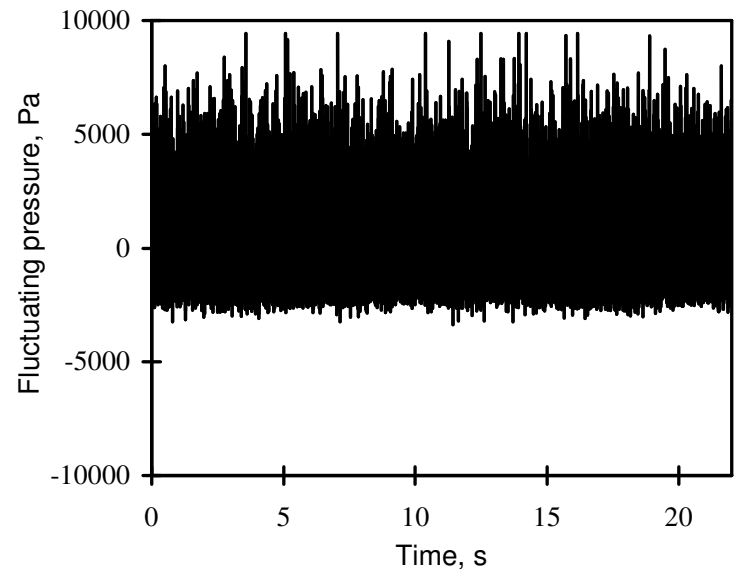

a) At the point immediately before the step

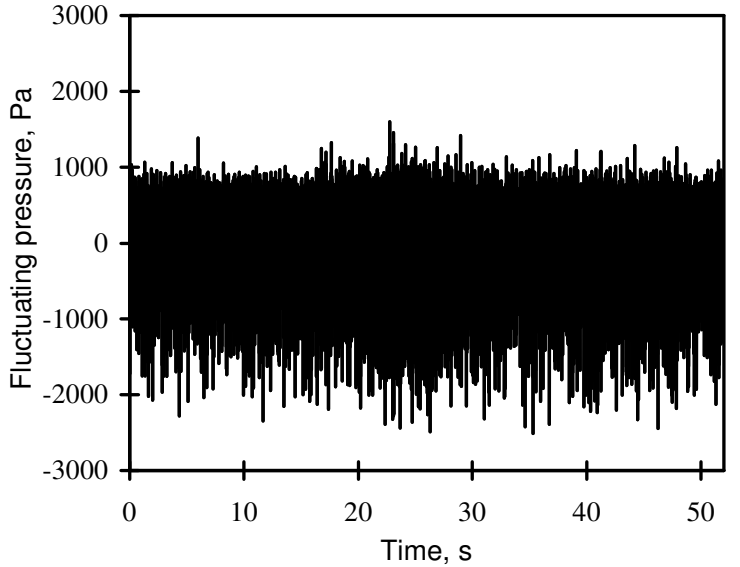

b) At the point further from the step

Fig 6 Fluctuating pressure time histories upstream of forward-facing step at Mach 2.0 


\section{Time History Considerations.}

The effect of PDF skewness and differences between the left and right PDF tails are clearly demonstrated in the fluctuating pressure time histories. At the point immediately before the forward-facing step where skewness was $\lambda=1.2$ for the first data example considered in previous section, positive pressure values are much higher than negative values (see Fig. 6,a). The difference between the highest positive and negative time history peaks is about three times. That is what is reflected by the difference of the PDF tails in Fig. 5,b. The right tail of the experimental PDF extends up to $9500 \mathrm{~Pa}$ whereas the same probability of occurrence at the left tail is for negative pressure values around $-3000 \mathrm{~Pa}$.

For the second data example with negative skewness $\lambda=-1.3$ at the point further upstream of the forwardfacing step, negative pressure values in the time history are up to $-2500 \mathrm{~Pa}$ (Fig. 6,b) whereas positive peaks are not higher than $1500 \mathrm{~Pa}$. Consequently, the PDF tails also exchanged their behavior when the left tail has become longer than the right tail (Fig. 5,d). Thus the effect of positive pressure prevailing at the point immediately in front of the forward-facing step then changes to domination of negative pressure at the point further upstream of the step.

\section{Skewness and Kurtosis at Different Distances from Forward-Facing Steps.}

Further analysis of random fluctuating pressure records has been undertaken in terms of skewness and kurtosis values. This particularly addressed the issue of how non-Gaussian behavior depends on the aircraft speed and the point of measurement in the vicinity of forward-facing steps, i.e. dependence on the distance from the steps. Kurtosis and skewness values obtained for one subsonic and four supersonic flight conditions are given in Tables 2 and 3.

The results are rounded at just one decimal place as the objective was to show tendencies in kurtosis/skewness behavior rather than to give their precise values. The bold font in the tables emphasizes the most important non-Gaussian deviations including the reverse in the skewness sign discussed in Section IV.B. Results for two step plates of the same height but located at the front and rear of the aircraft fuselage are presented in the adjacent rows.

For each of the flight conditions (aircraft speed), two step heights (4 and $7 \mathrm{~mm}$ ) were tested at the port side as explained in Section II. The results obtained for each height comprise separate sections of Tables 2 and 3 corresponding to the aircraft speed given in the first column. Different step heights produced different numerical results for skewness and kurtosis, although similar tendencies in the non-Gaussian behavior were observed for both heights at the same speed.

The kurtosis values for the measurements with no discontinuities are shown in the last column of Table 2. Kurtosis changes moderately with the speed increase but does not depend on location (front or rear of the fuselage). Thus, for the results with no steps it is sufficient to present just one kurtosis value for each of the speeds for immediate comparison with $4 \mathrm{~mm}$ and $7 \mathrm{~mm}$ step data located in the table further to the left. There was no need in showing skewness of smooth surface data in Table 3 because, for all speeds, the skewness with no discontinuities is close to zero. This should be kept in mind when assessing skewness results for steps.

As could be expected, kurtosis and skewness analyses show that intensity of non-Gaussian behavior for the surface with discontinuities depends on the upstream distance from the step. Data from transducers located at $112 \mathrm{~mm}$ before the step indicate no notable differences compared to the smooth surface data. The skewness values are nearly zero and kurtosis values were close to those of the smooth surface, though a bit smaller in the supersonic regime. This suggests that beyond a particular point upstream of the forward facing step, the effect of the step does not further influence the non-Gaussian parameters of the fluctuating pressure. The situation changes as the proximity to the step increases.

The next available transducer at $56 \mathrm{~mm}$ upstream of forward-facing step showed strong non-Gaussian behavior in terms of skewness. For example, at the highest supersonic speed of Mach 2.0, the skewness values were large and, what is most interesting, opposite in sign for different step heights of $4 \mathrm{~mm}$ (for which skewness was $\lambda=0.7$ ) and $7 \mathrm{~mm}(\lambda=-0.8)$. A similar effect was also observed for Mach 1.8. Kurtosis was changing as well when proceeding from 112 to $56 \mathrm{~mm}$ distances. For the $4 \mathrm{~mm}$ step at Mach 2.0, the kurtosis reached a value of 4.4, which is notably different from $\gamma=3.8$ obtained from the data with no discontinuities at this speed. For the Mach 1.8 speed the kurtosis even changed both to larger and smaller values: $\gamma=2.5$ for the $4 \mathrm{~mm}$ step and $\gamma=6.2$ for the $7 \mathrm{~mm}$ step.

Closer to the step, at $32 \mathrm{~mm}$ in front of it, the differences for two step heights were significant as well. At Mach 2.0, the fluctuating pressure data from the larger step $(7 \mathrm{~mm}$ ) was actually Gaussian with no skewness (changed from $\lambda=-0.8$ at $56 \mathrm{~mm}$ ) and very mild kurtosis $(\gamma=3.2)$. The latter is much less than for the smooth surface data 
and also a significant drop from $\gamma=4.0$ at $56 \mathrm{~mm}$ for the front location. However, for the lower step of $4 \mathrm{~mm}$, the strongest non-Gaussian behavior occurred in contrast to the above. The skewness $\lambda=-1.4$ and kurtosis $\gamma=6.5$ were the highest of all measurements analyzed. At lower speeds of Mach 1.8 and Mach 1.6, the results at the same $32 \mathrm{~mm}$ distance from the step were close to Gaussian.

For the next two transducers at $21 \mathrm{~mm}$ and $12 \mathrm{~mm}$ from the step, the pressure fluctuation signals for all supersonic speeds behaved close to the Gaussian model with some mild non-Gaussian deviations emerging at $6 \mathrm{~mm}$ from the step. Positive skewness values ranging from 0.4 to 0.6 were observed in this region. Then, immediately before the step (1 mm upstream), a strong non-Gaussian behavior was observed again. For the Mach 2.0 speed and $4 \mathrm{~mm}$ step height, the skewness returned from the negative value (it had at some distance) to the positive value $(\lambda=0.8)$ now close to the step.

Overall, the Mach 2.0/4 mm rows in Table 3 show that the skewness distribution as a function of distance from the step may exhibit an oscillatory pattern. The transducers at distances of $1 \mathrm{~mm}$ and $56 \mathrm{~mm}$ upstream represent positive peaks in skewness pattern, with a negative peak at $32 \mathrm{~mm}$ between them (the kurtosis values were large at all three points with the skewness peaks). For the $7 \mathrm{~mm}$ step and the same Mach 2.0 speed, there is one positive skewness peak at $1 \mathrm{~mm}$ and one negative, now at $56 \mathrm{~mm}$ from the step. The skewness goes through zero between these two peaks at the $32 \mathrm{~mm}$ location.

The same behavior has been observed for the $7 \mathrm{~mm}$ step for lower supersonic speed of Mach 1.8 and also for Mach 1.6 at the $4 \mathrm{~mm}$ step height. For the remaining two conditions (Mach 1.6/7mm and Mach 1.8/4mm) no negative skewness peaks were indicated by the available transducers. It is possible that they are still present somewhere between the 32 and $56 \mathrm{~mm}$ transducer locations, especially as further upstream one can see again an increase of positive skewness after it being around zero. If so, the spatial resolution was not small enough to detect negative skewness values.

There is no sign of an oscillatory pattern for skewness at the lowest supersonic speed of Mach 1.2 and subsonic regime Mach 0.75. The first and second sections of Table 3 show just a steady increase of skewness when approaching the forward-facing step from upstream. As for the kurtosis, at lower speeds of Mach 1.6 and Mach 1.2, the only point where kurtosis values were essentially higher $(\gamma=5.0$ and $\gamma=4.5)$ than for no-step data was at $1 \mathrm{~mm}$ in front of the $7 \mathrm{~mm}$ step. For the subsonic regime Mach 0.75, all kurtosis values upstream of the forward-facing step (except at $6 \mathrm{~mm}$ in front of the $7 \mathrm{~mm}$ step) were not higher than for the smooth surface data.

For both non-Gaussian parameters, kurtosis and skewness, an important observation is that, in almost all measurements, there is no sensible difference between the results obtained at the front and rear of the aircraft for a given step height. R.m.s values of the fluctuating pressure are different, but non-Gaussian parameters are not. Moreover, PDF curves for identical conditions at the front and at the rear were close to each other at both the PDF peak and tails. Thus, the general conclusion is that the non-Gaussian behavior of fluctuating pressure upstream of forward-facing step discontinuities is highly dependent upon distance from the step and the step height. The behavior is less dependent on aircraft speed, and shows no dependency on location on the fuselage.

\section{E. Results for Aft-Facing Step Discontinuities.}

Kurtosis and skewness values for positions downstream of an aft-facing step do not exhibit as strong a pattern of behavior with distance from the step and across flight regimes as for positions upstream of the forward-facing step. For brevity, tabular data is not shown. Kurtosis values are generally lower than those of the smooth surface data. At Mach 0.75 and at Mach 1.2 speeds, the kurtosis increases in value moderately with increasing distance downstream of the step. This tendency is reduced at Mach 1.6, and is not evident at Mach 2.0.

Skewness values are mainly within $-0.2<\lambda<0.2$, that is a level which can be considered indistinguishable from the Gaussian behavior. Only at the location closest to the aft-facing step greater positive and negative magnitudes up to $\lambda= \pm 0.5$ are evident. As with forward-facing step discontinuities, for a given step height and distance from the step, skewness and kurtosis downstream of the aft-facing step exhibit similar magnitudes in front and rear fuselage locations. 
Table 2. Kurtosis values for fluctuating pressure signals upstream of forward-facing steps

\begin{tabular}{|c|c|c|c|c|c|c|c|c|c|c|}
\hline \multirow{3}{*}{$\begin{array}{l}\text { Aircraft } \\
\text { speed }\end{array}$} & \multicolumn{9}{|c|}{ Surface with forward-facing step discontinuity } & \multirow{3}{*}{$\begin{array}{l}\text { Smooth } \\
\text { surface }\end{array}$} \\
\hline & \multirow{2}{*}{$\begin{array}{c}\text { Location } \\
\text { on } \\
\text { fuselage }\end{array}$} & \multirow[b]{2}{*}{$\begin{array}{l}\text { Step } \\
\text { height }\end{array}$} & \multicolumn{7}{|c|}{ Distance upstream of the step } & \\
\hline & & & $\begin{array}{l}112 \\
\mathrm{~mm}\end{array}$ & $\begin{array}{c}56 \\
\mathrm{~mm}\end{array}$ & $\begin{array}{c}32 \\
\mathrm{~mm}\end{array}$ & $\begin{array}{c}21 \\
\mathrm{~mm}\end{array}$ & $\begin{array}{c}12 \\
\mathrm{~mm}\end{array}$ & $\begin{array}{c}6 \\
\mathrm{~mm}\end{array}$ & $\begin{array}{c}1 \\
\mathrm{~mm}\end{array}$ & \\
\hline \multirow{4}{*}{$\begin{array}{l}\text { Mach } \\
0.75\end{array}$} & front & $4 \mathrm{~mm}$ & 4.2 & 3.8 & 3.4 & 3.4 & 3.3 & 3.2 & 3.6 & \multirow{4}{*}{4.2} \\
\hline & rear & $4 \mathrm{~mm}$ & 4.1 & 3.5 & 3.4 & 3.4 & 3.3 & 3.2 & 3.4 & \\
\hline & front & $7 \mathrm{~mm}$ & 4.0 & 3.5 & 3.4 & 3.4 & 3.3 & 4.6 & 4.0 & \\
\hline & rear & $7 \mathrm{~mm}$ & 3.9 & 3.3 & 3.3 & 3.3 & 3.2 & 4.6 & no data & \\
\hline \multirow{4}{*}{ Mach 1.2} & front & $4 \mathrm{~mm}$ & 3.8 & 3.4 & 3.3 & 3.4 & 3.5 & 3.4 & 3.6 & \multirow{4}{*}{4.1} \\
\hline & rear & $4 \mathrm{~mm}$ & 3.3 & 3.2 & 3.3 & 3.3 & 3.3 & 3.3 & 3.7 & \\
\hline & front & $7 \mathrm{~mm}$ & 3.6 & 3.3 & 3.3 & 3.4 & 3.4 & 3.9 & 4.4 & \\
\hline & rear & $7 \mathrm{~mm}$ & 3.3 & 3.2 & 3.2 & 3.2 & 3.4 & 3.7 & 4.6 & \\
\hline \multirow{4}{*}{ Mach 1.6} & front & $4 \mathrm{~mm}$ & 3.6 & 3.8 & 3.2 & 3.2 & 3.3 & 3.4 & 3.8 & \multirow{4}{*}{4.0} \\
\hline & rear & $4 \mathrm{~mm}$ & 3.5 & 3.1 & 3.1 & 3.0 & 3.1 & 3.2 & 3.9 & \\
\hline & front & $7 \mathrm{~mm}$ & 3.6 & 3.6 & 3.3 & 3.4 & 3.6 & 3.8 & 5.0 & \\
\hline & rear & $7 \mathrm{~mm}$ & 3.6 & 3.6 & 3.2 & 3.2 & 3.5 & 3.7 & 5.0 & \\
\hline \multirow{4}{*}{ Mach 1.8} & front & $4 \mathrm{~mm}$ & 3.7 & 2.6 & 3.4 & 3.1 & 3.2 & 3.3 & 3.7 & \multirow{4}{*}{3.8} \\
\hline & rear & $4 \mathrm{~mm}$ & 3.5 & 2.5 & 4.3 & 2.9 & 3.1 & 3.2 & 3.9 & \\
\hline & front & $7 \mathrm{~mm}$ & 3.7 & 5.7 & 3.3 & 3.3 & 3.7 & 3.8 & 5.1 & \\
\hline & rear & $7 \mathrm{~mm}$ & 3.5 & 6.8 & 3.2 & 3.2 & 3.6 & 3.7 & 5.1 & \\
\hline \multirow{4}{*}{ Mach 2.0} & front & $4 \mathrm{~mm}$ & 3.6 & 4.3 & 7.3 & 3.0 & 3.1 & 3.3 & 5.3 & \multirow{4}{*}{3.8} \\
\hline & rear & $4 \mathrm{~mm}$ & 3.3 & 4.4 & 5.7 & 2.9 & 3.0 & 3.2 & 5.4 & \\
\hline & front & $7 \mathrm{~mm}$ & 3.6 & 4.1 & 3.3 & 3.3 & 3.6 & 3.7 & 3.8 & \\
\hline & rear & $7 \mathrm{~mm}$ & 3.3 & 3.1 & 3.2 & 3.2 & 3.5 & 3.6 & 4.0 & \\
\hline
\end{tabular}

Table 3. Skewness values for fluctuating pressure signals upstream of forward-facing steps

\begin{tabular}{|c|c|c|c|c|c|c|c|c|c|}
\hline \multirow[b]{2}{*}{$\begin{array}{c}\text { Aircraft } \\
\text { speed }\end{array}$} & \multirow{2}{*}{$\begin{array}{c}\text { Location } \\
\text { on } \\
\text { fuselage }\end{array}$} & \multirow[b]{2}{*}{$\begin{array}{l}\text { Step } \\
\text { height }\end{array}$} & \multicolumn{7}{|c|}{ Distance upstream of the step } \\
\hline & & & $\begin{array}{l}112 \\
\mathrm{~mm}\end{array}$ & $\begin{array}{c}56 \\
\mathrm{~mm}\end{array}$ & $\begin{array}{c}32 \\
\mathrm{~mm}\end{array}$ & $\begin{array}{c}21 \\
\mathrm{~mm}\end{array}$ & $\begin{array}{c}12 \\
\mathrm{~mm}\end{array}$ & $\begin{array}{c}6 \\
\mathrm{~mm}\end{array}$ & $\begin{array}{c}1 \\
\mathrm{~mm}\end{array}$ \\
\hline \multirow{4}{*}{$\begin{array}{l}\text { Mach } \\
0.75\end{array}$} & front & $4 \mathrm{mmm}$ & $\overline{0}$ & 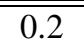 & 0.3 & $\overline{0.4}$ & $\overline{0.5}$ & $\overline{0.4}$ & 0.3 \\
\hline & rear & $4 \mathrm{~mm}$ & 0.1 & 0.2 & 0.4 & 0.5 & 0.5 & 0.4 & 0.4 \\
\hline & front & $7 \mathrm{~mm}$ & 0.1 & 0.2 & 0.4 & 0.5 & 0.4 & 0.0 & 0.7 \\
\hline & rear & $7 \mathrm{~mm}$ & 0.1 & 0.3 & 0.4 & 0.5 & 0.4 & -0.1 & no data \\
\hline \multirow{4}{*}{ Mach 1.2} & front & $4 \mathrm{~mm}$ & 0 & 0.1 & 0.4 & 0.5 & 0.6 & 0.6 & 0.5 \\
\hline & rear & $4 \mathrm{~mm}$ & 0 & 0.2 & 0.4 & 0.6 & 0.6 & 0.5 & 0.6 \\
\hline & front & $7 \mathrm{~mm}$ & 0 & 0.2 & 0.4 & 0.5 & 0.5 & 0.3 & 0.9 \\
\hline & rear & $7 \mathrm{~mm}$ & 0 & 0.2 & 0.4 & 0.5 & 0.5 & 0.3 & 1.0 \\
\hline \multirow{4}{*}{ Mach 1.6} & front & $4 \mathrm{~mm}$ & -0.1 & -0.6 & 0.2 & 0.4 & 0.5 & 0.5 & 0.7 \\
\hline & rear & $4 \mathrm{~mm}$ & 0 & -0.6 & 0.1 & 0.3 & 0.4 & 0.4 & 0.7 \\
\hline & front & $7 \mathrm{~mm}$ & 0.3 & 0.2 & 0.2 & 0.4 & 0.4 & 0.3 & 1.1 \\
\hline & rear & $7 \mathrm{~mm}$ & 0.3 & 0.1 & 0.2 & 0.4 & 0.4 & 0.4 & 1.1 \\
\hline \multirow{4}{*}{ Mach 1.8} & front & $4 \mathrm{~mm}$ & -0.1 & 0.3 & 0.0 & 0.3 & 0.5 & 0.5 & 0.6 \\
\hline & rear & $4 \mathrm{~mm}$ & 0 & 0.4 & -0.2 & 0.3 & 0.4 & 0.4 & 0.7 \\
\hline & front & $7 \mathrm{~mm}$ & -0.1 & -0.6 & 0.1 & 0.3 & 0.4 & 0.3 & 1.1 \\
\hline & rear & $7 \mathrm{~mm}$ & 0 & -1.2 & 0.1 & 0.3 & 0.3 & 0.3 & 1.2 \\
\hline \multirow{4}{*}{ Mach 2.0} & front & $4 \mathrm{~mm}$ & -0.1 & 0.7 & -1.4 & 0.1 & 0.4 & 0.4 & $\overline{0.7}$ \\
\hline & rear & $4 \mathrm{~mm}$ & 0 & 0.7 & -1.3 & 0.1 & 0.3 & 0.3 & 0.8 \\
\hline & front & $7 \mathrm{~mm}$ & -0.1 & -0.9 & 0.1 & 0.3 & 0.4 & 0.3 & 1.2 \\
\hline & rear & $7 \mathrm{~mm}$ & 0 & -0.7 & 0 & 0.2 & 0.3 & 0.3 & 1.2 \\
\hline
\end{tabular}




\section{Analytical Approximation of Experimental PDFs}

Non-Gaussian behavior of fluctuating pressure PDFs is evident from the results presented in the previous section. The difference between experimental probability distributions and the Gaussian analytical distribution (1) was principal and significant. Hence, no appropriate approximation can be achieved by use of the Gaussian model. Other models, flexible towards various skewness and kurtosis values, are necessary. It means that, additionally to the mean value $m$ and variance $\sigma^{2}$, present in the Gaussian function (1), there should be two more parameters in the approximating non-Gaussian analytical PDF.

Classical theory of statistics suggests three possible solutions to the above problem: Pearson family of probability density functions (each for a particular non-Gaussian case), Johnson system of some nonlinear transformations of the standard Gaussian variable, and expansions into series in the Gram-Charlier or Edgeworth form $[13,14]$.

\section{A. Pearson and Johnson Distributions.}

To find out which particular distribution of these two families is applicable, one needs to put his experimental skewness and kurtosis values on special diagrams (see Ref. [13]) showing areas occupied by different analytical PDF functions in the system of $\lambda-\gamma$ coordinates. Then, properties of the selected distribution function should be checked against those required in the problem under consideration and, if not in agreement, the analytical distribution should be declined.

Particularly, for many of the Pearson and Johnson distributions, the variable is confined in the limited interval with bounds normally defined from some physical reasoning (for example, the variable can take positive values only). Fluctuating pressure random process is obviously not of such kind. Any pressure values can occur, i.e. the PDF argument should have unlimited scope from $-\infty$ to $\infty$.

Only the Type IV distribution from the Pearson family

$$
P_{I V}(u)=C\left(a^{2}+u^{2}\right)^{-\zeta} \exp [-b \arctan (u / a)]
$$

and the $S_{u}$ type from the Johnson system

$$
P_{S u}(u)=a\left[(u-\varepsilon)^{2}+\eta^{2}\right]^{-1 / 2} \exp \left\{-0.5\left[b+a \log \left\{(u-\varepsilon) / \eta+\sqrt{(u-\varepsilon)^{2} / \eta^{2}+1}\right\}\right]^{2}\right\} / \sqrt{2 \pi}
$$

meet the condition of unlimited variable $u$.

Thus, if an experimental pressure fluctuation record produced skewness $\lambda^{*}$ and kurtosis $\gamma^{*}$ values, which are in the scope of the above two PDF types, then Eqs (7) and (8) can be used as approximating functions. Such an example is the fluctuating pressure data with $\lambda^{*}=0.75$ and $\gamma^{*}=5.35$ at the point of $1 \mathrm{~mm}$ upstream of the forward facing step of $4 \mathrm{~mm}$ height at the Mach 2.0 vehicle speed.

On the other hand, if the $\left(\lambda^{*}, \gamma^{*}\right)$ pair fell into the region of other Pearson or Johnson distributions, then this kind of analytical approximation cannot be used. At the same location and the aircraft speed as above but for another step height of $7 \mathrm{~mm}$, the skewness $\lambda *=1.2$ and kurtosis $\gamma^{*}=3.9$ values obtained are indicative of other Pearson and Johnson distributions, Type I and $S_{B}$ correspondingly. These two types are not applicable as the range of their arguments is not unlimited.

Although approximation functions (7) and (8) have necessary four parameters to make them flexible in terms of mean value $m$, standard deviation $\sigma$, skewness $\lambda$, and kurtosis $\gamma$, it is not an easy matter to actually find $C, a, b$, and $\zeta$ of the Pearson Type IV or $a, b, \varepsilon$ and $\eta$ of the Johnson $S_{u}$ type for the specified $m, \sigma, \lambda$, and $\gamma$ values. Unfortunately, there is no analytical solution for this problem. Some comments are present in [14] about possible numerical procedures and tables available. However, it is a serious obstacle that skewness and kurtosis are involved in these analytical PDFs in implicit form via other parameters. Generally, it is concluded in Ref. [14] that "probability density functions (7) and (8) are very difficult to handle comfortably in practice". 


\section{B. Gram-Charlier Series.}

Compared to the Pearson or Johnson distributions the expansion into truncated series is much easier to handle and it has been widely used in the area of dynamics and vibrations $[15,16]$. An equation for the Gram-Charlier series is as follows

$$
P_{G C}(u)=\frac{1}{\sigma \sqrt{2 \pi}} \exp \left(-\frac{\tilde{u}^{2}}{2}\right)\left[1+\frac{\lambda}{6} \tilde{u}\left(\tilde{u}^{2}-3\right)+\frac{\gamma-3}{24}\left(\tilde{u}^{4}-6 \tilde{u}^{2}+3\right)\right]
$$

where $\tilde{u}=(u-m) / \sigma$ is a standardized non-dimensional variable. To find $P_{G C}(u)$ for the given argument $u$, the standardized variable $\tilde{u}$ should be calculated first and, then, the result substituted into Eq (9). This actually gives the Gram-Charlier PDF for the real (not standardized) variable $u$ as a function with four parameters $m, \sigma, \lambda$, and $\gamma$.

The first exponential component in Eq (9) resembles the Gaussian distribution (1) but then it is multiplied by the second component involving skewness $\lambda$ and kurtosis $\gamma$ parameters in a convenient explicit form. Thus, if one needs to approximate a PDF with the given skewness and kurtosis values, it remains only to substitute them into Eq (9) and the function $P_{G C}(u)$ obtained will possess the necessary skewness and kurtosis according to the above definitions of these parameters by Eqs (2) in Section III.

However, the behavior of function (9) may become contradictory to basic properties of probability density functions because the Gram-Charlier series are truncated when establishing this analytical model. Either Gaussian or non-Gaussian, the PDF must always be positive but the Gram-Charlier function (9) may lead to inappropriate negative values for some argument ranges and certain kurtosis and skewness values.

It has been found $[17,18]$ that the most critical situation is when the kurtosis value $\gamma$ is less than 3 . If so, the last summand in Eq (9) obtains negative coefficient and will increase without limit with the increase of argument u. It means that negative values by $\mathrm{Eq}(9)$ will be inevitable for large arguments, i.e. at the distribution tails from a certain starting value to infinity.

Although the fluctuating pressure data measured belong to the opposite case of $\gamma>3$, another condition occurs when the same last summand in $\mathrm{Eq}$ (9) may again be negative, now within the intervals $[-\sigma \sqrt{3+\sqrt{6}},-\sigma \sqrt{3-\sqrt{6}}]$ and $[\sigma \sqrt{3-\sqrt{6}}, \sigma \sqrt{3+\sqrt{6}}]$. In this case, the negative sections of $P_{G C}(u)$ look as valleys located on both sides of the PDF near the argument values $|u-m|=\sqrt{3} \sigma$. For skewed distributions this effect appears for smaller kurtosis values and the valleys are not symmetrical and different in width (see below in Fig. 8,b). It should be pointed out that the valleys may still exist even if there is no negative $P_{G C}(u)$ values (see Figs. 7,c and 8,a). This is also not appropriate in the problem under consideration as all experimental PDFs were unimodal with no additional peaks in the tail areas.

Overall the Gram-Charlier expansion can be regarded for convenience in usage and, thus, it has been implemented for approximating the fluctuating pressure data. The results obtained were checked for meaningless negative values and unnecessary valleys. If any faulty PDFs are observed they were ruled out.

\section{Hermite Polynomial Transformation.}

To overcome drawbacks of the Gram-Charlier model, the idea of functional transformation of the Gaussian random variable (as in the Johnson system) has been further developed by Winterstein [19]. This method employs Hermite polynomials like the Gram-Charlier expansion. However, now it avoids erroneous negative distribution values by applying Hermite functions not to the PDF but to the time history itself or, more precisely, to a nonlinear function used for transformation of time histories.

A random variable or process $u$ with a non-Gaussian PDF can be standardized and centralized with respect to its $m$ and $\sigma$ values. The resulting non-dimensional non-Gaussian variable $\tilde{u}$ is supposed to be equal to a functional transformation of some Gaussian variable $v$ with this transformation taken in the form of $k$-term Hermite series

$$
\frac{u-m}{\sigma}=\tilde{u}=f(v)=q\left[v+\sum_{n=3}^{k} h_{n} H e_{n-1}(v)\right] .
$$


If the series are truncated at the order of $k=4$, the above transformation from Gaussian to non-Gaussian variables looks as follows

$$
\tilde{u}=f(v)=q\left[v+h_{3}\left(v^{2}-1\right)+h_{4}\left(v^{3}-3 v\right)\right]
$$

Coefficient $q$ is for scaling purposes only but two other parameters, $h_{3}$ and $h_{4}$, influence the PDF shape of the standardized variable $\tilde{u}$ obtained as a result of transformation. By adjusting these two parameters, the specified skewness $\lambda^{*}$ and kurtosis $\gamma^{*}$ values can be achieved for the non-Gaussian variable $u$. Analytical relations between $h_{3}, h_{4}$, and $\lambda^{*}, \gamma^{*}$ were given in $\operatorname{Ref}[19]$

$$
h_{3}=\lambda^{*} /\left\{4+2 \sqrt{1+1.5\left(\gamma^{*}-3\right)}\right\}, \quad h_{4}=\left\{\sqrt{1+1.5\left(\gamma^{*}-3\right)}-1\right\} / 18 \text {. }
$$

The scale coefficient has been also found [19]

$$
q=1 / \sqrt{1+2 h_{3}^{2}+6 h_{4}^{2}}
$$

from the condition that the standardized intermediate variable $\tilde{u}$ must have unit variance.

The probability density function $P_{2}(y)$ of some monotonic one-to-one functional transformation $y=f(x)$ of random variable $x$ with the given probability density $P_{1}(x)$ is defined [20] as

$$
P_{2}(y)=\left[P_{1}(x) /\left|\frac{d f(x)}{d x}\right|\right]_{x=f^{-1}(y)}
$$

where $x$ in the right hand side must be replaced by $g^{-1}(y)$ that is an inverse function to the function $f(x)$ describing the transform applied to initial random variable $x$.

Thus, to construct the non-Gaussian analytical PDF by a functional transform (11) based on Hermite polynomials, the solution of Eq (11) for $v$ in terms of $u$ is required. For the 4-th order Hermite series, this solution has been given by Winterstein [19] in analytical form

$$
v(u)=\left[\sqrt{\xi^{2}(u)+c}+\xi(u)\right]^{1 / 3}-\left[\sqrt{\xi^{2}(u)+c}-\xi(u)\right]^{1 / 3}-a
$$

where

$$
\xi(u)=1.5 b[a+(u-m) /(q \sigma)]-a^{3}, b=1 /\left(3 h_{4}\right), a=b h_{3}, c=\left(b-1-a^{2}\right)^{3} .
$$

Variable $V$ as a function of variable $u$ given by Eq (15) should be substituted into the following equation for the probability density function

$$
P_{H}(u)=\frac{1}{q \sigma \sqrt{2 \pi}}\left[\exp \left(-\frac{x^{2}}{2}\right) /\left(3 h_{4} x^{2}+2 h_{3} x+1-3 h_{4}\right)\right]_{x=v(u)}
$$

obtained according to the general equation (14) describing the PDF transformation.

The Hermite polynomial transform method provides an approximation of non-Gaussian PDFs in a way described by Eqs (12), (13), (15), (16) and (17). The probability density function obtained $P_{H}(u)$ can be drawn and compared with experimental data, as is later shown for the boundary layer fluctuating pressure measurements. However, 
approximation of experimental PDFs normally implies not only fitting to data but also some further action with the analytical PDF constructed.

Hence, an important additional criterion of successful approximation is how suitable the obtained PDF is for subsequent analytical manipulations. The ability to easily manipulate the Gaussian distribution law is what made it so powerful and widely used. There are numerous efficient solutions for various problems based on the assumption that the PDF is Gaussian. The Gram-Charlier series (9) is also good from this point of view, making it the most frequently used non-Gaussian PDF.

As for Hermite polynomial transform, Equations (12), (13), (15), (16) and (17) describing it are complex and do not lend themselves to further analysis in analytical form, for instance, if there is a need to integrate. In this regard, it would be useful to develop a model that avoids erroneous negative values as Hermite polynomials method but still remains similar in form to the Gaussian law as Gram-Charlier series.

\section{Symmetric Piecewise-Gaussian Approximation.}

As an alternative to the above methods, a piecewise-Gaussian model has been proposed [21]. The idea of this approach is to construct non-Gaussian PDFs from a few sections of Gaussian distributions (1), each with different parameters. This was done in such a way as to avoid negative values of the probability density and to ensure its unimodality. The model suggested is more flexible in data fitting than the simple Gaussian distribution, but its use is no more complicated than that of the Gaussian model because the piecewise-Gaussian approximation simply entails application of the Gaussian model (1) several times.

A symmetrical $(\lambda=0)$ centralized $(m=0)$ non-Gaussian distribution with variable kurtosis value $\gamma$ has been composed [21] from two quasi-Gaussian exponential functions $X_{1,2}(u)=\exp \left[-u^{2} /\left(2 \sigma_{1,2}^{2}\right)\right]$ with different variances $\sigma_{1}^{2}$ and $\sigma_{2}^{2}$

$$
P_{S}(u)=\left\{\begin{array}{lrr}
Q \exp \left[-\left(u-\sigma_{2}+v\right)^{2} /\left(2 \sigma_{2}^{2}\right)\right], & \text { at } & u \leq-v \\
Q\left\{\exp \left[-u^{2} /\left(2 \sigma_{1}^{2}\right)\right]+H\right\}, & \text { at } & -v<u \leq v \\
Q \exp \left[-\left(u+\sigma_{2}-v\right)^{2} /\left(2 \sigma_{2}^{2}\right)\right], & \text { at } & u>v
\end{array}\right.
$$

In the $[-v, v]$ interval the distribution obtained is represented by the first function $X_{1}(u)$. If this middle section is shifted vertically by the value $H=\left|X_{2}\left(\sigma_{2}\right)-X_{1}(v)\right|$, it will meet two symmetric tails taken from the second function, $X_{2}(u)$, and shifted horizontally by the value $\left|\sigma_{2}-v\right|$. All sections of the piecewise-Gaussian PDF were joined in such a way that conditions of continuity for the function $P_{s}(u)$ itself and for its derivative were preserved. Since the quasi-Gaussian tails were shifted horizontally only, they never acquire negative values and thus retain this property of the Gaussian model (1). This is an advantage of the piecewise-Gaussian distribution over the GramCharlier series.

There are five parameters, $Q, H, \sigma_{1}, \sigma_{2}$, and $v$ in the PDF equation (18) but they are not independent and can all be expressed [21] in terms of the experimental standard deviation $\sigma$ and one more specifically introduced non-Gaussian parameter $\beta$. The latter allows to impart the necessary kurtosis value $\gamma^{*}$ to the function $P_{S}(u)$. The relation between $\beta$ and $\gamma^{*}$ has been established in the form of a nonlinear algebraic equation given in Ref [21] which can be resolved for $\beta$ with the prescribed kurtosis value $\gamma^{*}$. Then all parameters in Eq (18) can be found and the piecewise-Gaussian PDF with necessary kurtosis value $\gamma^{*}$ constructed.

It appeared that for the aircraft sidewall surface with no discontinuities when the fluctuating pressure PDF is symmetric (see Section IV.A above), the distribution tails are wider than those that could be provided by the approximating function (18). To comply with these conditions observed in the experimental data, a new type of piecewise-Gaussian model has been developed.

Two basic quasi-Gaussian exponential functions $X_{1,2}(u)$ that made up the piecewise-Gaussian Type 1 distribution (18) were multiplied now by additional factors which are inversely proportional to $\sigma_{1}$ and $\sigma_{2}$. This action changed the equations for piecewise-Gaussian parameters and, particularly, the relation between $\beta$ and $\gamma^{*}$. A range of possible $\gamma^{*}$ values was extended and analytical PDFs with the tails wider than in the probability density (18) were obtained. 
The equations for this symmetric piecewise-Gaussian Type 2 are as follows. The centralized analytical PDF with the standard deviation $\sigma$

$$
P_{S 2}(u, \sigma, \beta)= \begin{cases}\frac{C Y}{\sigma \sqrt{2 \pi}}\left[\exp \left(-\frac{u^{2}}{2 \sigma^{2} B^{2}}\right)+H\right] & \text { at }|u| \leq \beta B \sigma \\ \frac{C}{\sigma \sqrt{2 \pi}} \exp \left\{-\frac{[|u|+\sigma B(Y-\beta)]^{2}}{2 \sigma^{2} Y^{2} B^{2}}\right\} & \text { at }|u|>\beta B \sigma\end{cases}
$$

has four parameters $C, B, Y$, and $H$ that are expressed through $\beta$

$$
\begin{gathered}
H(\beta)=\exp \left(-\frac{1}{2}\right) \frac{1}{Y}-\exp \left(-\frac{\beta^{2}}{2}\right), C(\beta)=\frac{1}{Y} \sqrt{\frac{\pi}{2}}[D(\beta) B(\beta)], Y(\beta)=\sqrt{\frac{1}{\beta} \exp \left[\left(\beta^{2}-1\right) / 2\right]}, \\
B(\beta)=\sqrt{D}\left\{\beta^{3} \frac{H}{3}+\Phi(\beta)-\beta \exp \left(-\frac{\beta^{2}}{2}\right)+\left[\sqrt{\frac{\pi}{2}}-\Phi(1)\right]\left(2 Y^{2}-2 \beta Y+\beta^{2}\right)+Y(2 \beta-Y) \exp \left(-\frac{1}{2}\right)\right\}^{-\frac{1}{2}} \\
D(\beta)=\beta H+\Phi(\beta)+\sqrt{\frac{\pi}{2}}-\Phi(1), \quad \Phi(\beta)=\int_{0}^{\beta} \exp \left(-\frac{\omega^{2}}{2}\right) d \omega .
\end{gathered}
$$

The nonlinear equation for $\beta$

$$
\begin{gathered}
\beta^{5} \frac{H(\beta)}{5}-\beta\left(\beta^{2}+3\right) \exp \left(-\frac{\beta^{2}}{2}\right)+\exp \left(-\frac{1}{2}\right)\left[4 Y^{4}-12 Y^{3}(Y-\beta)+6 Y^{2}(Y-\beta)^{2}-4 Y(Y-\beta)^{3}\right] \\
+3 \Phi(\beta)+\left[\sqrt{\frac{\pi}{2}}-\Phi(1)\right]\left[3 Y^{4}+6 Y^{2}(Y-\beta)^{2}+(Y-\beta)^{4}\right]=\frac{D(\beta)}{B^{4}(\beta)} \gamma^{*}
\end{gathered}
$$

can be solved numerically.

\section{E. General Asymmetric Piecewise-Gaussian Model.}

On the basis of the symmetric piecewise-Gaussian model (18), a non-symmetrical type with the increased kurtosis and variable skewness has been developed [10,21]. Asymmetry of the experimental centralized PDF means that the position of the distribution peak does not coincide with the mean value $m$. If an argument of the PDF peak (which is called the mode value) is shifted, for instance, to the left as in Fig. 5,a, then the PDF is skewed and the skewness is positive (opposite case of negative skewness is shown in Fig. 5,c).

Suppose that on the left and on the right of the mode value $\mu$ there are two sections of different symmetric piecewise-Gaussian PDFs (18) shifted by $\mu$ horizontally with respect to the coordinate origins

$$
P_{A}(u)=\left\{\begin{array}{lll}
Q_{A} \exp \left\{\left[u-\mu-\sigma_{2 L}+v_{L}\right]^{2} /\left(2 \sigma_{2 L}^{2}\right)\right\}, & \text { at } \quad u \leq \mu-v_{L} \\
Q_{A}\left\{\exp \left[-(u-\mu)^{2} /\left(2 \sigma_{1 L}^{2}\right)\right]+H_{L}\right\}, & \text { at } \quad \mu-v_{L}<u \leq \mu \\
Q_{A}\left\{\exp \left[-(u-\mu)^{2} /\left(2 \sigma_{1 R}^{2}\right)\right]+H_{R}\right\}, & \text { at } \quad \mu<u \leq \mu+v_{R} \\
Q_{A} \exp \left\{-\left[u-\mu+\sigma_{2 R}-v_{R}\right]^{2} /\left(2 \sigma_{2 R}^{2}\right)\right\}, & \text { at } \quad u>\mu+v_{R} .
\end{array}\right.
$$

Comparison of the second and third lines in this equation suggests that smoothness of the function (22) at the point $U=\mu$ can be ensured only if $H_{L}=H_{R}$. It means that both left and right halves of the asymmetric model (each representing a symmetric Type 1 or 2 ) must have the same value of the symmetric non-Gaussian parameter $\beta$. However the latter is not the only non-Gaussian characteristic. 
After satisfying all necessary conditions, the parameters present in Eq (22) are expressed via the experimental standard deviation $\sigma$, kurtosis-related parameter $\beta$ and one more parameter $\alpha$ which can be related to skewness. Thus, instead of one equation, like Eq (21), for finding $\beta$ for the given $\gamma^{*}$ in the symmetric case, there will be a set of two equations in $\alpha$ and $\beta$. When the solution is found, the $\alpha$ and $\beta$ values obtained will impart the given skewness $\lambda *$ and kurtosis $\gamma^{*}$ to the piecewise-Gaussian model (22). Expressions for these two equations and other details regarding the formulation of the asymmetric piecewise-Gaussian distribution $P_{A}(u)$ may be found in Ref [21].

\section{Comparison of Non-Gaussian Models with Experimental Distributions}

\section{A. Smooth Surface with no Discontinuities (Symmetric PDF Case).}

The Gram-Charlier series, the Hermite polynomial transform, and the symmetric piecewise-Gaussian Type 1 and Type 2 models were exercised for the smooth surface data considered in Section IV.A. For the symmetric case Eqs (9) and (17) simplify after substitution of zero skewness $\lambda$. When constructed with the same kurtosis value $\gamma$ as found in experimental data, the symmetric Gram-Charlier model (dotted curve in Fig. 7,a) gave a little improvement compared to the Gaussian approximation of the same data (Fig. 7,b). If one tries to make the Gram-Charlier PDF tails wider by increasing the kurtosis in $\mathrm{Eq}$ (9), the analytical distribution changes dramatically and in an unacceptable manner (dotted curve in Fig. 7,c) which was predicted above in Section V.B.

The Type 1 piecewise-Gaussian distribution (18) presented by the dotted curve in Fig. 7,d was closer to the experimental PDF than the Gaussian model shown in Fig. 7,b for comparison. The improvement was about the same as that provided by the Gram-Charlier approximation in Fig. 7,a. The precision of the Type 1 approximation was limited by the fact that the kurtosis value for this piecewise-Gaussian model cannot be larger than a certain boundary value. To increase the boundary kurtosis value and to make the PDF tails wider, the Type 2 modification of the symmetrical piecewise-Gaussian model was applied according to Eqs (19-21). The result obtained is shown by the dotted curve in Fig. 7,e and it captures the behavior of the experimental PDF tails, albeit at some compromise to the PDF middle section.

Finally, the Hermite polynomials transform approximation is seen in Fig. 7,f to agree very well with the experimental distribution. For the symmetric PDF case with skewness $\lambda$ set to zero, equations (15-17) simplify because $h_{3}$ and $a$ become zero. Even in this form, however, the Hermite polynomial PDF is complicated and, hence, difficult to handle in further analytical manipulations where the fluctuating pressure probability density might be involved. This is considered a drawback compared with the piecewise-Gaussian model.

\section{B. Fluctuating Pressure in Front of Forward-Facing Steps (Skewed PDF case).}

As for the above symmetric PDF case, the same three models have been implemented but now in the most general form considered in Section V to take into account not only kurtosis but the skewness parameter as well. Experimental PDFs of fluctuating pressure immediately $(1 \mathrm{~mm})$ in front of the $7 \mathrm{~mm}$ forward-facing step were shown by solid curves in Fig. 5, a (area near the PDF peak) and in Fig. 5,b (PDF tails) in comparison with the Gaussian approximation (dotted curves). Asymmetry of the experimental PDF is obvious on both plots whereas the Gaussian distribution is, of course, symmetrical.

The skewness and kurtosis values found from the experimental data and used for analytical models were $\lambda *=1.2$ and $\gamma^{*}=4.0$ (see the last row in Tables 2 and 3). The Gram-Charlier approximation showed some shift of the peak to the left (dotted curve in Fig. 8,a) but to the lesser extent than in the experimental PDF (solid curve in Fig. 8,a). However, this was accompanied by improper changes of the PDF for positive arguments. A valley has appeared for intermediate pressure values and it was followed by an unnecessary increase in the PDF, making all approximation on the right from the PDF peak inappropriate (Fig. 8,a).

On the left from the PDF peak, erroneous behavior is also apparent in the distribution tail plot (Fig. 8,b). Compared to the right PDF slope, a valley at the left appears farther from the mean value and for smaller probabilities. Moreover, the bottom of the valley erroneously extends to negative probability values that should not be the case for a PDF model. As negative values cannot be shown on the logarithmic plot, the Gram-Charlier dotted curve in Fig. 8,b breaks between -3000 and $-4000 \mathrm{~Pa}$. Thus, the difference between the experimental and analytical distributions is essential for both tails and, hence, approximation by the Gram-Charlier model is unsatisfactory. 


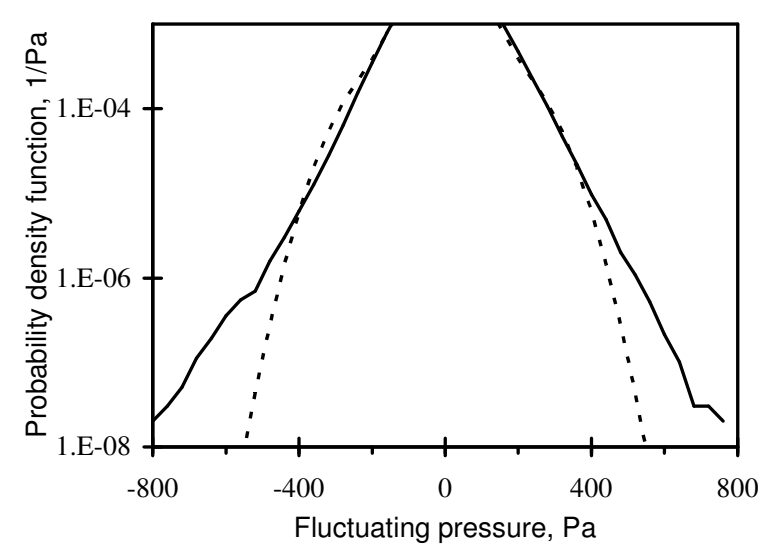

a) Gram-Charlier series with kurtosis $\gamma=\mathbf{4 . 0}$

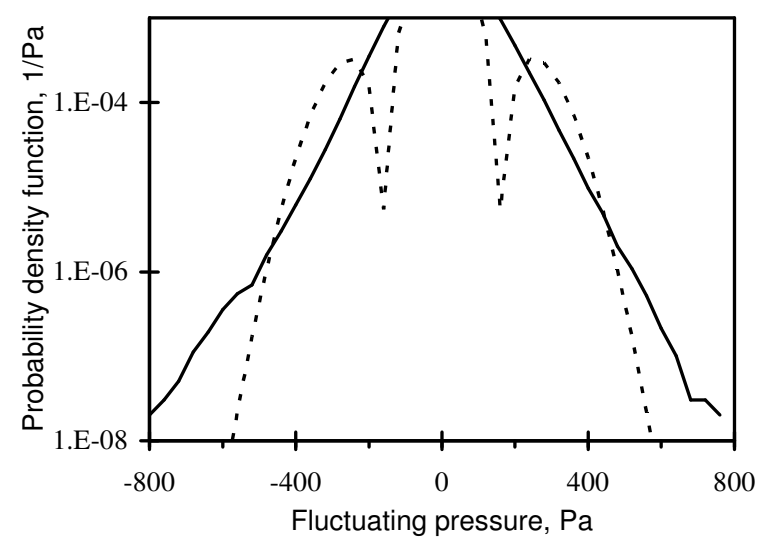

c) Gram-Charlier series with kurtosis $\gamma=\mathbf{7 . 0}$

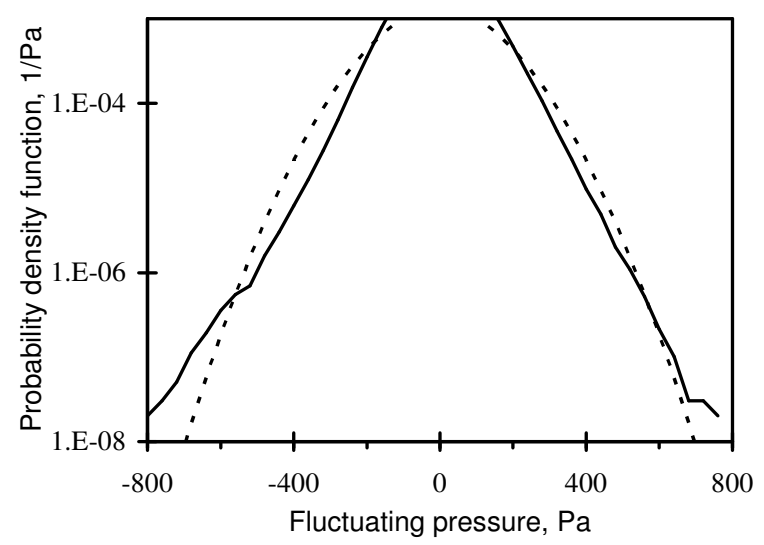

e) Piecewise-Gaussian model Type 2

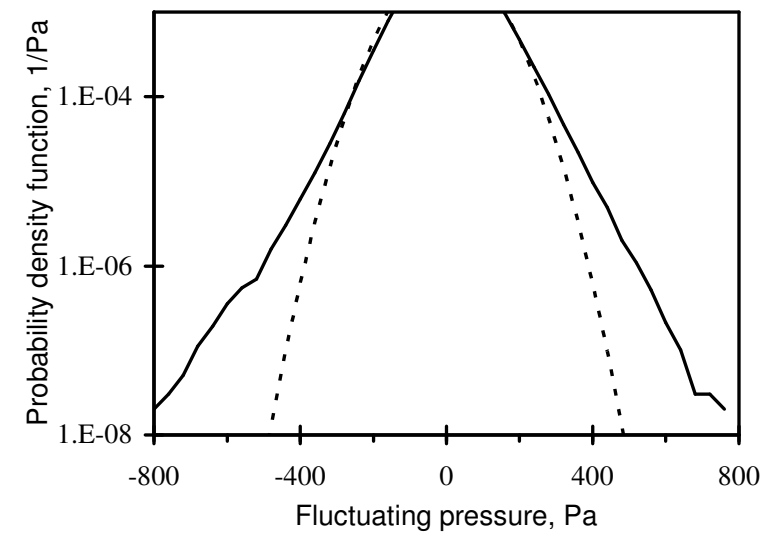

b) Gaussian model

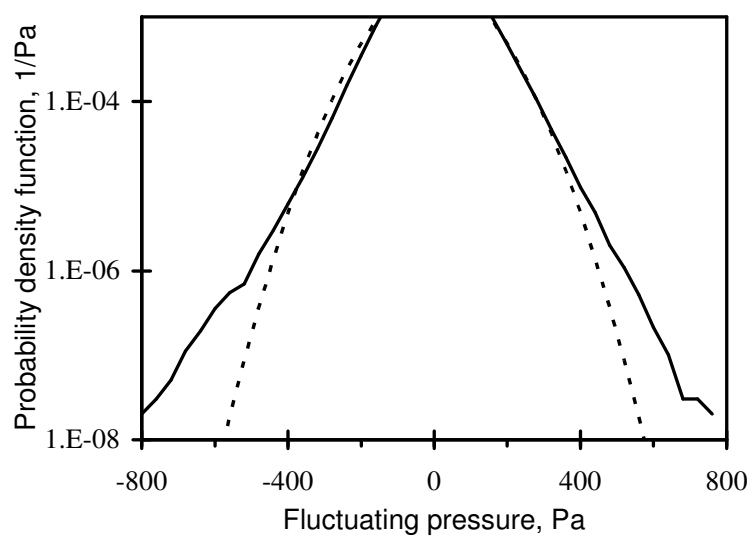

d) Piecewise-Gaussian model Type 1

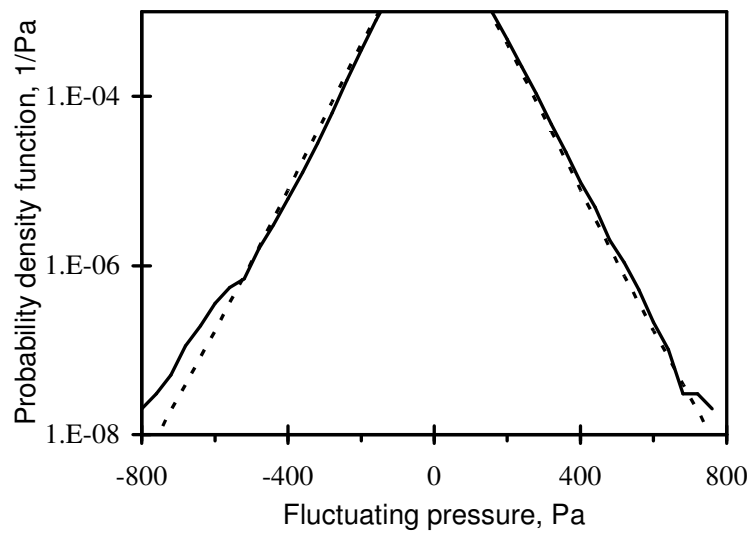

f) Hermit polynomial transform

Fig. 7 Fluctuating pressure PDF tails for smooth sidewall condition at a subsonic speed

(flight data - solid curve, analytical approximations - dotted curves) 


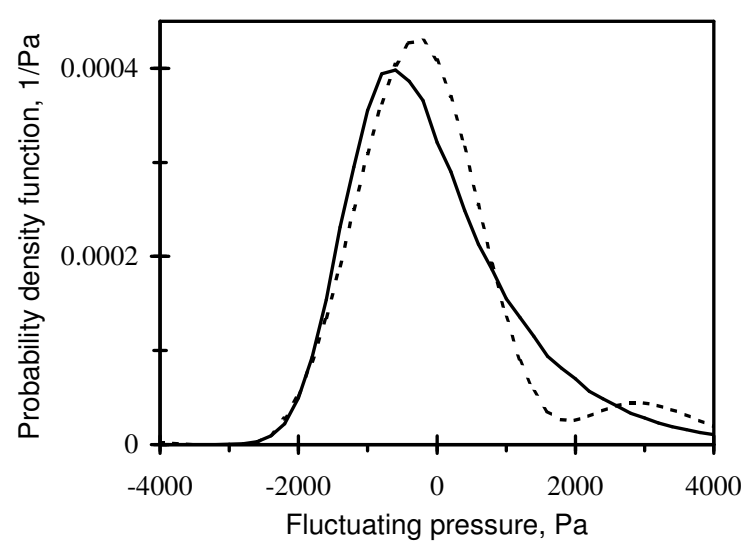

a) Gram-Charlier model of PDF peak

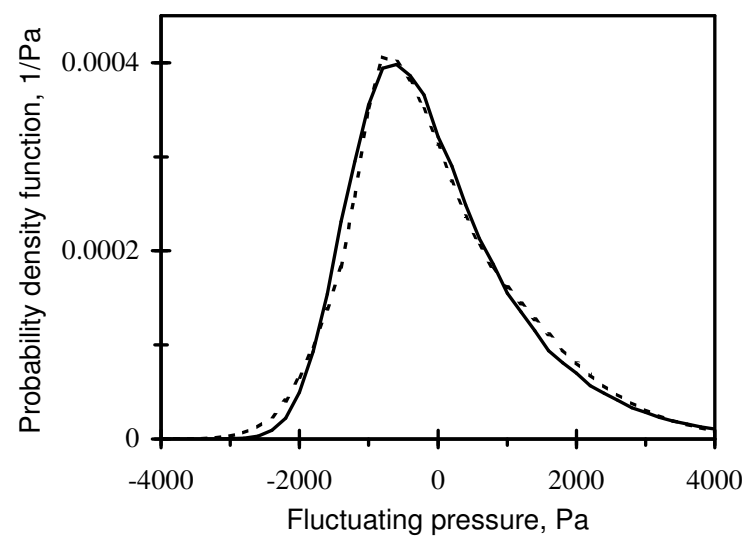

c) Piecewise-Gaussian model of PDF peak

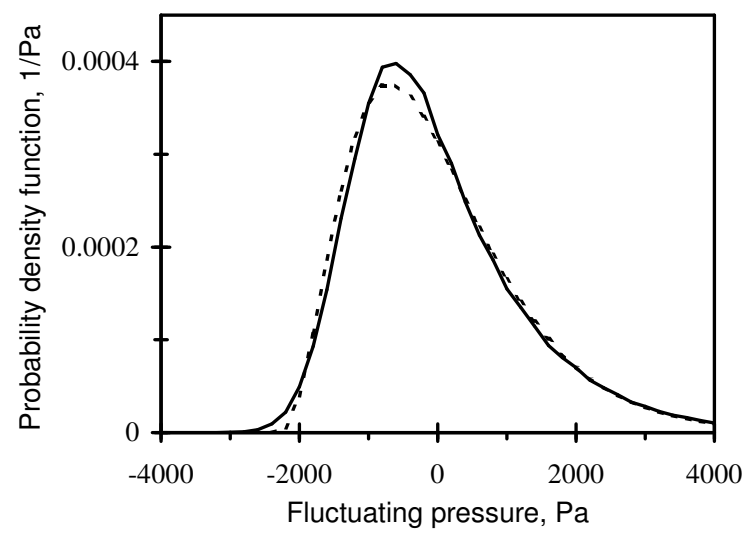

e) Hermit polynomial model of PDF peak

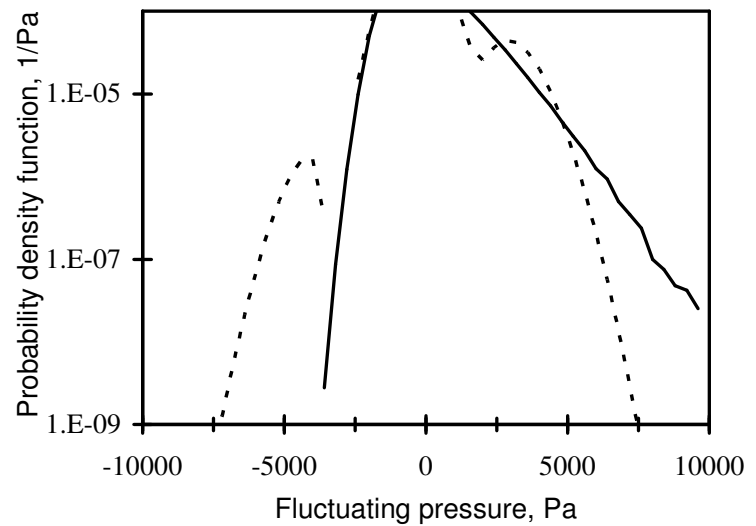

b) Gram-Charlier model of PDF tails

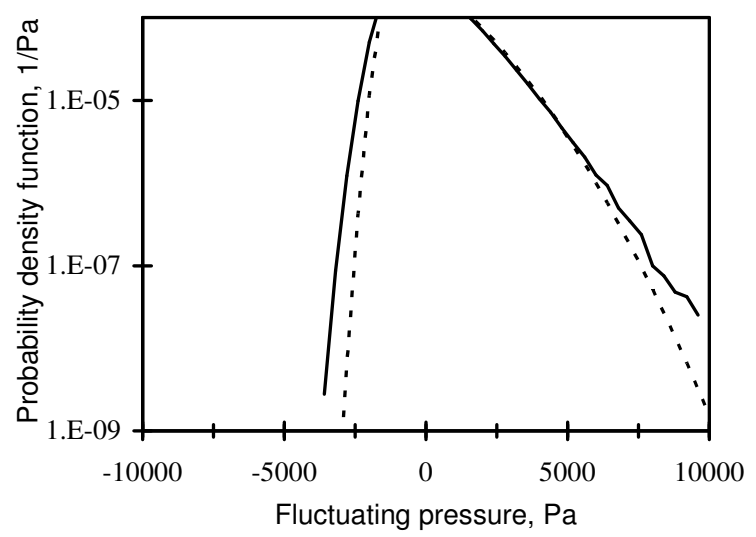

d) Piecewise-Gaussian model of PDF tails

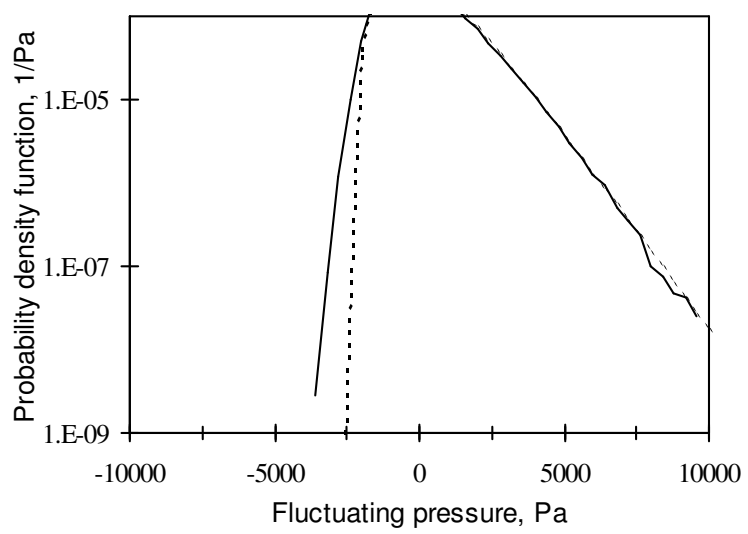

f) Hermit polynomial model of PDF tails

Fig. 8 Fluctuating pressure PDFs before step (7 mm height) for supersonic speed Mach 2.0

(flight data - solid curve, analytical approximations - dotted curves) 
The more sophisticated non-symmetric piecewise-Gaussian and Hermite polynomial models were much more successful. Figure 8,c shows that the PDF peak was captured nearly perfectly using the piecewise-Gaussian model, and was also modeled well using the Hermite polynomial transformation model (see Fig. 8,e). There are some minor differences in the tails, but both models reflected the asymmetrical shape well. The piecewise-Gaussian model worked slightly better at the left tail (Fig. 8,d) whereas the Hermite polynomial model was more precise at the right tail (Fig. 8,f).

\section{Conclusions}

The turbulent boundary layer fluctuating pressures measured on the exterior of a supersonic transport aircraft Tu-144LL were investigated. Data was acquired and analyzed for the regular smooth fuselage sidewall, and for sidewalls with forward-facing and aft-facing step discontinuities under various subsonic and supersonic flight conditions. Since the behavior of the fluctuating pressure probability density (Gaussian or non-Gaussian) affects further analysis of acoustic radiation and structural integrity, the data processing concentrated on determination of the PDF and calculation of skewness and kurtosis characteristics.

The influence of measurement location on the fuselage and the aircraft speed, the surface step height and distance from the step were studied and the vast majority of fluctuating pressure signals appeared to be non-Gaussian processes both for subsonic and supersonic flight conditions. The most important differences from the Gaussian PDF were noted at the distribution tails. For the smooth wall condition, skewness was near zero like for a Gaussian process but the kurtosis appeared to be much larger. The latter was in correspondence with the PDF tails discovered to be wider and longer than those of the Gaussian model.

For the sidewall with discontinuities the experimental non-Gaussian PDFs of fluctuating pressure in front of forward-facing steps were asymmetrical both in the middle and tail sections with skewness values up to 1.4 , that is a very strong deviation from the Gaussian behavior. The kurtosis values at many locations also increased essentially compared to the smooth wall measurements. For higher supersonic speeds, the skewness spatial pattern upstream of the forward-facing step included positive and negative values at certain distances from the step.

Overall the non-Gaussian behavior of fluctuating pressure signals appeared to be highly dependent upon the distance from the step and the step height, less dependent on aircraft speed, and not dependent on the fuselage location. The results obtained at the locations near the front and the rear of the aircraft under the same flight condition were very close in all characteristics analyzed. The latter also applies to the data measured downstream of the aft facing steps, however the spatial pattern is different, the non-Gaussian parameters are lower and any trends in them are subtle.

To approximate the experimental PDFs several probability distribution models were evaluated including the Pearson and Johnson distribution families, the Gram-Charlier series, Hermite polynomial transform, and piecewiseGaussian approximations. Application of the Gram-Charlier series appeared to be problematic both for smooth and stepped exterior wall conditions. Low precision and non-physical negative probability values were obtained for certain flight conditions. The Hermite polynomial transformation approximation modeled both the smooth wall (symmetric) and forward-facing step (asymmetric) PDF behaviors well. However, its mathematical form is not conducive to further analytical manipulation with the PDF.

In the above context, the advantage of the piecewise-Gaussian model is that it is constructed from two or four Gaussian sections adjusted at the boundary points but having different parameters. The subsequent use of piecewiseGaussian analytical distributions requires nothing more than recurring manipulation with the Gaussian probability function. From a practical standpoint, this allows previously developed Gaussian solutions for structural dynamics and fatigue prediction problems to be employed in situations in which the PDF is non-Gaussian. The piecewiseGaussian approximations generally worked well providing precision comparable with that of the Hermite polynomials model. A new piecewise-Gaussian type was developed which allowed for higher values of kurtosis observed in experimental fluctuating pressure measurements.

\section{References}

[1] Frendi, A., "Coupling between a supersonic turbulent boundary layer and a flexible structure," AIAA Journal, Vol. 35, No. 1, 1997, pp. 58-66.

[2] Chase, D. M., "Modeling the wavenumber-frequency spectrum of turbulent boundary layer wall pressure," Journal of Sound and Vibration, Vol. 70, No. 1, 1980, pp. 29-67.

[3] Efimtsov, B. M., "Characteristics of the field of turbulent wall pressure fluctuations at large Reynolds numbers", Soviet Fhysics-Acoustics, Vol. 28, No. 4, 1982, pp.289-292.

[4] Graham, W. R., "A comparison of models for the wavenumber-frequency spectrum of turbulent boundary layer pressures," Journal of Sound and Vibration, Vol. 206, No. 4, 1997, pp. 541-565. 
[5] Efimtsov, B. M., Kozlov, N. M., Kravchenko, S. V., and Andersson, A. O., "Wall pressure-fluctuation spectra at small forward-facing steps," Proceedings of the 5th AIAA/CEAS Aeroacoustics Conference, AIAA-99-1964, Bellevue, WA, 1999.

[6] Efimtsov, B. M., Golubev, A. Y., Rizzi, S. A., Andersson, A. O., Rackl, R. G., and Andrianov, E. V., "Influence of small steps on wall pressure fluctuation spectra measured on TU-144LL flying laboratory," Proceedings of the 8th AIAA/CEAS Aeroacoustics Conference, AIAA-2002-2605, Breckenridge, CO, 2002.

[7] Wirsching, P. H., and Light, M. C., "Fatigue under wide band random stresses", ASCE Journal of the Structural Division", Vol. 106, No 7, 1980, pp. 1593-1601.

[8] Spottswood, S. M., and Wolfe, H. F., "Comparing fatigue life estimates using experimental and spectral density based probability distributions", Journal of Aircraft, Vol. 39, No 3, 2002, pp. 493-498.

[9] Bishop, N.W.M. and Sherratt, F., "A theoretical solution for the estimation of rainflow ranges from power spectral density data," Fatigue and Fracture of Engineering. Materials and Structures, Vol. 13, No. 1990, pp. 311-326.

[10] Steinwolf, A. and White, R. G., "Probability density functions of acoustically-induced strains in experiments with composite panels", AIAA Journal, Vol. 35, No. 12, 1997, pp. 1853-1861.

[11] Rizzi, S. A., Rackl, R. G., and Andrianov, E. V., "Flight test measurements from the Tu-144LL structure/cabin noise experiment," NASA TM-2000-209858, January 2000.

[12] Rizzi, S. A., Rackl, R. G., and Andrianov, E. V., "Flight test measurements from the Tu-144LL structure/cabin noise follow-on experiment," NASA TM-2000-209859, February 2000.

[13] Hann, M., and Shapiro, S. S, Statistical Models in Engineering, John Wiley, New York, 1967.

[14] Kendall, M., and Stuart, A., The Advanced Theory of Statistics, Distribution Theory, Vol. 1, Charles Griffin and Co., London, 1977.

[15] Crandall, S. H., " Non-Gaussian closure for random vibration of non-linear oscillators," International Journal of NonLinear Mechanics, Vol. 15, 1980, pp. 303-313.

[16] Melzer, H. J., and Schueller, G. J., " On the reliability of flexible structures under non-normal loading processes," Proceedings of IUTAM Symposium on Random Vibration and Reliability, Academie-Verlag, Frankfurt/Oder, 1983, pp. 73-83.

[17] Barton, D. E. and Dennis, K. E, "The conditions under which Gram-Charlie and Edgeworth curves are positive definite and unimodal", Biometrika, Vol. 39, 1952, pp. 425-427.

[18] Steinwolf, A., Ferguson, N. S., and White, R. G, "Variations in steepness of the probability density function of beam random vibration", European Journal of Mechanics A/Solids, Vol. 19, 2000, pp. 319-341.

[19] Winterstein S. R., "Nonlinear vibration models for extremes and fatigue", ASCE Journal of Engineering Mechanics, Vol. 114, 1988, pp. 1772-1790.

[20] Bendat, J. S., Nonlinear Systems, Techniques and Applications, John Wiley, New York, 1998.

[21] Steinwolf, A., "Analysis and approximation of probability distribution of vehicle random vibrations with consideration of kurtosis and skewness values", Proceedings of 5th International Conference on Recent Advances in Structural Dynamics, Vol. 2, Southampton, 1994, pp. 785-794. 